\title{
PROPERTIES OF HIGH RANK SUBVARIETIES OF AFFINE SPACES
}

\author{
DAVID KAZHDAN AND TAMAR ZIEGLER
}

\begin{abstract}
We use tools of additive combinatorics for the study of subvarieties defined by high rank families of polynomials in high dimensional $\mathbb{F}_{q}$-vector spaces. In the first, analytic part of the paper we prove a number properties of high rank systems of polynomials. In the second, we use these properties to deduce results in Algebraic Geometry, such as an effective Stillman conjecture over algebraically closed fields, an analogue of Nullstellensatz for varieties over finite fields, and a strengthening of a recent result of $[5$. We also show that for $k$-varieties $\mathbb{X} \subset \mathbb{A}^{n}$ of high rank any weakly polynomial function on a set $\mathbb{X}(k) \subset k^{n}$ extends to a polynomial.
\end{abstract}

\section{INTRODUCTION}

Let $k$ be a field. For an algebraic $k$-variety $\mathbb{X}$ we write $X(k):=\mathbb{X}(k)$. To simplify notations we often write $X$ instead of $X(k)$. In particular we write $V:=\mathbb{V}(k)$ when $\mathbb{V}$ is a vector space and write $k^{N}$ for $\mathbb{A}^{N}(k)$.

For a $k$-vector space $\mathbb{V}$ we denote by $\mathcal{P}_{d}(\mathbb{V})$ the algebraic variety of polynomials on $\mathbb{V}$ of degree $\leq d$ and by $\mathcal{P}_{d}(V)$ the set of polynomials functions $P: V \rightarrow k$ of degree $\leq d$. We always assume that $d<|k|$, so the restriction map $\mathcal{P}_{d}(\mathbb{V})(k) \rightarrow$ $\mathcal{P}_{d}(V)$ is a bijection. For a family $\bar{P}=\left\{P_{i}\right\}$ of polynomials on $\mathbb{V}$ we denote by $\mathbb{X}_{\bar{P}} \subset \mathbb{V}$ the subscheme defined by the ideal generated by $\left\{P_{i}\right\}$ and by $X_{\bar{P}}$ the set $\mathbb{X}_{P}(k) \subset V$. We will not distinguish between the set of affine $k$-subspaces of $\mathbb{V}$ and the set of affine subspaces of $V$ since for an affine $k$-subspace $\mathbb{W} \subset \mathbb{V}$, the $\operatorname{map} \mathbb{W} \rightarrow \mathbb{W}(k)$ is a bijection.

In the introduction we consider only the case of hypersurfaces $\mathbb{X} \subset \mathbb{V}$ and provide an informal outline of main results. Precise definitions appear in the next section.

Definition 1.1. Let $P$ be a polynomial of degree $d$ on a $k$-vector space $V$.

(1) We denote by $\tilde{P}: V^{d} \rightarrow k$ the multilinear symmetric form associated with $P$ defined by $\tilde{P}\left(h_{1}, \ldots, h_{d}\right):=\Delta_{h_{1}} \ldots \Delta_{h_{d}} P: V^{d} \rightarrow k$, where $\Delta_{h} P(x)=$ $P(x+h)-P(x)$.

(2) The $\operatorname{rank} r(P)$ is the minimal number $r$ such that $P$ can be written in the form $P=\sum_{i=1}^{r} Q_{i} R_{i}$, where $Q_{i}, R_{i}$ are polynomials on $V$ of degrees $<d$.

The second author is supported by ERC grant ErgComNum 682150. 
(3) We define the non-classical rank (nc-rank) $r_{n c}(P)$ to be the rank of $\tilde{P}$.

(4) A polynomial $P$ is $m$-universal if for any polynomial $Q \in \mathcal{P}_{d}\left(k^{m}\right)$ of degree $d$ there exists an affine map $\phi: k^{m} \rightarrow V$ such that $Q=P \circ \phi$.

(5) We denote by $\mathbb{X}_{P} \subset \mathbb{V}$ the hypersurface defined by the equation $P(v)=0$ and by $\mathbb{X}_{P}^{\text {sing }}$ the singular locus of $\mathbb{X}_{P}$.

(6) $s(P):=\operatorname{codim}_{\mathbb{X}_{P}}\left(\mathbb{X}_{P}^{\text {sing }}\right)$.

Remark 1.2. (1) If $\operatorname{char}(k)>d$ then $r(P) \leq r_{n c}(P)$.

(2) In low characteristic it can happen that $P$ is of high rank and $\tilde{P}$ is of low rank, for example in characteristic 2 the polynomial $P(x)=\sum_{1<i<j<k<l \leq n} x_{i} x_{j} x_{k} x_{l}$ is of rank $\sim n$, but of nc-rank 3, see [8], [24], [29].

Remark 1.3. Let $\mathcal{F}_{Q}$ be the set of all affine maps $\phi: k^{m} \rightarrow V$ such that $Q=P \circ \phi$. We later show that in the case when $r_{n c}(P) \gg 1$, the size of the set $\mathcal{F}_{Q}$ is essentially independent of the choice of $Q \in \mathcal{P}_{d}\left(k^{m}\right)$.

We now turn to the applications.

1.0.1. Sections of high rank varieties.

Theorem 1.4 (Acc). There exists $C=C(d)$ with the following property. For any field $k$ which is either algebraically closed or finite field, any $k$-vector space $V$ and any polynomial $P \in \mathcal{P}_{d}(V)$ of nc-rank $>C m^{C}$ the following hold:

(1) Any polynomial $Q \in \mathcal{P}_{d}\left(k^{m}\right)$ is m-universal.

(2) Let $A f f_{m}(\mathbb{V})$ be the variety of affine maps $\phi: \mathbb{A}^{m} \rightarrow \mathbb{V}$ and $\tilde{\kappa}_{P}: A f f_{m}(\mathbb{V}) \rightarrow$ $\mathcal{P}_{d}\left(\mathbb{A}^{m}\right)$ be the algebraic map defined by $\tilde{\kappa}_{P}(\phi):=P \circ \phi$. Then all fibers of $\tilde{\kappa}_{P}$ are algebraic varieties of the same dimension.

(3) The map $\tilde{\kappa}_{P}: A f f_{m}(\mathbb{V}) \rightarrow \mathcal{P}_{d}\left(\mathbb{A}^{m}\right)$ is flat.

Remark 1.5. (1) It is easy to see that $s(P) \leq 2 r(P)$.

(2) In the case when $k$ is an algebraically closed field of characteristic 0 it is shown in [27] that $r(P) \leq d ! s(d)$.

(3) We can show the existence of $\epsilon=\epsilon(d)>0$ such that $r_{n c}(P) \leq \epsilon s^{\epsilon}(P)$ for polynomials of degree $d$.

Corollary 1.6. There exist $C=C(d)$ such that for any field $k$ which is either algebraically closed or finite, a k-vector space $V$, any polynomial $P \in \mathcal{P}_{d}(V)$ such that $s(\tilde{P}) \geq C m^{C}$ is $m$-universal.

Question 1.7. Does there exist $\delta=\delta(d)$ such that $r(P) \leq \delta s(P)^{\delta}$ ?

Remark 1.8. The analogous result holds for a system $\bar{P}$ of polynomials.

1.0.2. A strengthening of the main Theorem from [5]. In [5] authors show that any non-trivial Zariski-closed condition on tensors that is functorial in the underlying vector space implies bounded rank. We show that the condition of being Zariskiclosed can be omitted. 
Theorem 1.9. Let $k$ be an algebraically closed field, $\mathcal{C}$ the category of finitedimensional affine $k$-vector spaces with morphisms being affine maps, let $\mathcal{F}_{d}$ be the contravariant endofunctor on $\mathcal{C}$ given by

$$
\mathcal{F}_{d}(V)=\{\text { Polynomials on } V \text { of degree } \leq d\},
$$

and let $\mathcal{G} \subset \mathcal{F}$ be a proper subfunctor. Then there exists $r$ such that $r_{n c}(P) \leq r$ for any finite-dimensional $k$-vector space $V$ and $P \in \mathcal{G}(V)$.

1.0.3. Extending weakly polynomial functions. Let $k$ be a field, $V$ a $k$-vector space and $X$ a subset of $V$. A function $f: X \rightarrow k$ is weakly polynomial of degree $\leq a$, if the restriction of $f$ on any affine subspace $L$ of $V$ contained in $X$ is a polynomial of degree $\leq a$.

Theorem 1.10. Let $k$ be a field which either an algebraically closed field, or a finite field such that $|k|>$ ad and let $X \subset V$ be a hypersurface defined by a polynomial of degree $d$ of sufficiently high nc-rank. Then any $k$-valued weakly polynomial function of degree $\leq a$ on $X$ is a restriction of a polynomial $F$ on $V$ of degree $\leq a$.

Remark 1.11. The main difficulty in a proof of Theorem 1.10 is the nonuniqueness of $F$ in the case when $a>d$.

1.0.4. Nullstellensatz over $\mathbb{F}_{q}$. We prove the following variant of Nullstellensatz for polynomials over $\mathbb{F}_{q}$.

Theorem 1.12. There exists $r(d)$ such that for any finite field $k=\mathbb{F}_{q}$, a $k$ vector space $V$ and a $k$-polynomial $P$ of degree $d$ and nc-rank larger than $r(d)$ the following holds: Any polynomial $R$ of degree $<q / d$ vanishing at all points $x \in X_{P}$ is divisible by $P$.

\section{Remark 1.13.}

(1) This result is a strengthening of the Proposition 9.2 from 8 in two ways:

(a) We show the independence of $r(d)$ on the degree of $R$, and

(b) The paper [4] shows the existence of polynomials $Q_{i}$ of bounded degrees such that $R(x)=\sum_{i=1}^{c} Q_{i}(x) P_{i}(x)$, for all $x \in \mathbb{X}(k)$, but does not show that $R$ is contained in the ideal generated by $\left\{P_{i}\right\}$.

(2) We outline ideas of proofs of Theorems 1.10, 1.12 at the beginning of corresponding sections.

(3) The quantitative bound on the rank in all proofs depend only on the bounds in Theorem 3.4. We conjectured that the bound on $r$ in Theorem 3.4 depends polynomially on $s$. This conjecture was proved in [26] (and in a slightly weaker form in [12]).

\section{The FORMUlation of RESUlts.}

We start with a series of definitions. 
Definition 2.1 (Rank). Let $k$ be field and $V$ a $k$-vector space.

(1) A tensor of degree $d$ on $V$ is a multilinear map $T: V^{d} \rightarrow k$.

(2) Let $P: V \rightarrow k$ be a polynomial of degree $d$. We define the $\operatorname{rank} r(P)$ as the minimal number $r$ such that $P$ can be written as a sum $P=\sum_{j=1}^{r} Q_{j} R_{j}$ where $Q_{j}, R_{j}$ are polynomials of degrees $<\operatorname{deg}(P)$ defined over $k 1$. We define the rank of a degree 1 polynomial to be infinite, and the rank of the zero polynomial to be 0 .

(3) For a polynomial $P: V \rightarrow k$ of degree $d$ we define $\tilde{P}\left(h_{1}, \ldots, h_{d}\right):=$ $\Delta_{h_{1}} \ldots \Delta_{h_{d}} P: V^{d} \rightarrow k$, where $\Delta_{h} P(x)=P(x+h)-P(x)$.

(4) We define the non-classical rank (nc-rank) $r_{n c}(P)$ to be the rank of $r(\tilde{P})$.

(5) For a $d$ tensor $P: V^{d} \rightarrow k$ we define the partition rank (p-rank) $\operatorname{pr}(P)$ as the minimal number $r$ such that $P$ can be written as a sum $P\left(x_{1}, \ldots, x_{d}\right)=$ $\sum_{J_{i} \subset[1, d], i \in[r]} Q_{j}\left(\left(x_{l}\right)_{l \in J_{i}}\right) R_{j}\left(\left(x_{l}\right)_{l \in J_{i}^{c}}\right)$ where $Q_{j}, R_{j}$ are degree $<d$ tensors.

(6) For any sequence $\bar{d}=\left(d_{1}, \ldots, d_{c}\right)$ we denote by $\mathcal{P}_{\bar{d}}(V)$ the space of families $\bar{P}=\left(P_{i}\right)_{1 \leq i \leq c}$ of polynomials such that $\operatorname{deg} P_{i} \leq d_{i}$ We define the rank $r(\bar{P})$ as the minimal rank of the polynomials $\sum_{\bar{a} \in k^{c} \backslash\{0\}} a_{i} P_{i}$. We denote $d:=\max d_{i}$. Define $r_{n c}(\bar{P}), \operatorname{pr}(\bar{P})$ similarly.

(7) For a family $\bar{P}=\left(P_{i}\right)_{1 \leq i \leq c}$ of polynomials on $\mathbb{V}$ we define the subscheme $\mathbb{X}_{\bar{P}} \subset \mathbb{V}$ by the system of equations $\left\{v \in V: P_{i}(v)=0,1 \leq i \leq c\right\}$, and define the rank of $\mathbb{X}_{\bar{P}}$ to be the rank of $\bar{P}$.

Remark 2.2. The rank $r(\bar{P})$ depends only on the linear span $L(\bar{P})$ of $\left(P_{i}\right)$.

The following statement is immediate.

Claim 2.3. Let $\bar{P}=\left(P_{i}\right)_{i=1}^{c}$ be a family of polynomials with $r(\bar{P}) \neq 0$. Then

(1) $\operatorname{dim}(L(\bar{P}))=c$.

(2) There exists a basis $Q_{i}^{j}$ in $L(\bar{P})$ such that

(a) $\operatorname{deg}\left(Q_{i}^{j}\right)=j$.

(b) For any $j$ the family $\bar{Q}^{j}=\left(Q_{i}^{j}\right)$ is of rank $\geq r(\bar{P})$.

Remark 2.4. Since the subscheme $\mathbb{X}_{\bar{P}}$ depends only on the space $L(\bar{P})$, we can (and will) assume that all the families $\bar{P}$ we consider satisfy the conditions of Claim 2.3 on $\bar{Q}$. We also may assume that $\bar{P}$ has no affine polynomials, since we can initially restrict to the locus of the affine polynomials.

Lemma 2.5. Let $P$ be a d-tensor. Then $r(P) \leq \operatorname{pr}(P) \leq 4^{d} r(P)$.

Proof. Let $P$ be a $d$-tensor. It is clear that $r(P) \leq \operatorname{pr}(P)$. We show that $\operatorname{pr}(P) \leq$ $4^{d} r(P)$. Let $k$ be a field with $|k| \geq d+2$ and write $G=\left(k^{\star}\right)^{d}$. For any $\bar{j}=$ $\left\{j_{1}, \ldots, j_{d}\right\}, j_{d} \leq d$ we denote by $\chi_{\bar{j}}: G \rightarrow k^{\star}$ the character $\bar{t} \rightarrow \prod_{i=1}^{d} t_{i}^{j_{i}}$ where $\bar{t}=\left(t_{1}, \ldots, t_{d}\right)$.

\footnotetext{
${ }^{1}$ This notion of rank is also known as Schmidt-rank in the analytic number theory literature or strength in the algebraic geometry literature.
} 
Let $V_{i}, 1 \leq i \leq d$ be $k$-vector spaces and $\mathcal{P}$ the space of polynomials $P$ : $V_{1} \times \ldots \times V_{d} \rightarrow k$ of degree $\leq d$. Let $J$ be the space of maps $\bar{j}$ from $[1, d]^{d} \rightarrow[1, d]$. For any $\bar{j} \in J$ we denote by $\mathcal{P}_{\bar{j}}$ the subspace of polynomials $P: V_{1} \times \ldots \times V_{d} \rightarrow k$ such that

$$
P\left(t_{1} v_{1}, \ldots, t_{d} v_{d}\right)=\prod_{i=1}^{d} t_{i}^{j_{i}} P\left(v_{1}, \ldots, v_{d}\right) .
$$

Since $|G|$ is prime to $\operatorname{char}(k)$ and $|k| \geq d+2$ we have a direct sum decomposition $\mathcal{P}=\oplus_{\bar{j} \in J} \mathcal{P}_{\bar{j}}$. We denote by $p_{\bar{j}}: \mathcal{P} \rightarrow \mathcal{P}_{\bar{j}}$ the projection

We write $\overline{1}=(1, \ldots, 1) \in J$ denote by $p_{d}: \mathcal{P} \rightarrow \mathcal{P}_{\overline{1}}$ the projection. For any $a, b, a+b=d$ we denote by $J_{a, b} \subset J \times J$ the set of pairs $\bar{j}_{a}, \bar{j}_{b}$ such that $\operatorname{supp}\left(\bar{j}_{a}\right) \cap \operatorname{supp}\left(\bar{j}_{b}\right)=\emptyset, \operatorname{deg}\left(\bar{j}_{a}\right)=a, \operatorname{deg}\left(\bar{j}_{b}\right)=b$.

For any polynomials $Q, R \in \mathcal{P}$ of degrees $a, b, a+b=d$ we write $P(Q, R)=$ $\sum_{\left(j_{1}, j_{2}\right) \in J_{a, b}} p_{j_{1}}(Q) p_{j_{2}}(R)$. We have that $p_{\overline{1}}(Q R)=P(Q, R)$, and therefore $\operatorname{pr}(P) \leq$ $4^{d} r(P)$.

\section{Definition 2.6.}

(1) For $m \geq 1$ and a $k$-vector space $V$, we denote by $\operatorname{Aff}_{m}(\mathbb{V})$ the algebraic variety of affine maps $\phi: \mathbb{A}^{m} \rightarrow \mathbb{V}$ and write $\operatorname{Aff}_{m}(V):=\operatorname{Aff}_{m}(\mathbb{V})(k)$.

(2) We define an algebraic morphism $\tilde{\kappa}_{\bar{P}}: \operatorname{Aff}_{m}(\mathbb{V}) \rightarrow \mathcal{P}_{\bar{d}}\left(\mathbb{A}^{m}\right)$ by $\tilde{\kappa}_{\bar{P}}(\phi):=$ $\bar{P} \circ \phi$, and denote by $\kappa_{\bar{P}}$ the corresponding map $\operatorname{Aff}_{m}(V) \rightarrow \mathcal{P}_{\bar{d}}\left(k^{m}\right)$.

Definition 2.7. A map $\kappa: M \rightarrow N$ between finite sets is $\epsilon$-uniform, where $\epsilon>0$, if for all $n \in N$ we have

$$
|| N|| \kappa^{-1}(n)|-| M|| \leq \epsilon|M| .
$$

Remark 2.8. In this paper we say that a bound $r(\bar{d}, m, t)$ is effective if

(1) For fixed $d:=\max _{i} d_{i}$, the bound is polynomial in $m, t, c$.

(2) The dependence on $d$ is doubly exponential.

The effective lower bounds for $r(\bar{d}, m, t)$ follow from the Conjecture 3.5 proven in [26].

Theorem 2.9. For any sequence $\bar{d}$ and any $m, t \geq 1$, there exists an effective bound $r(\bar{d}, m, t)$ such that for any finite field $k=\mathbb{F}_{q}$, a $k$-vector space $V$ and $a$ family $\bar{P} \in \mathcal{P}_{\bar{d}}(V)$ of nc-rank $\geq r(\bar{d}, m, t)$, the map $\kappa_{P}$ is $q^{-t}$ uniform.

To formulate Remark 1.3 precisely we introduce a number of additional definitions.

Definition 2.10. Fix an affine hypersurface $W \subset V$. We denote by $\mathbb{Z}_{\bar{P}}$ the variety of affine $m$-dimensional subspaces $L \subset X_{\bar{P}} \cap W$ and by $\mathbb{Y}_{\bar{P}} \subset \mathbb{Z}_{\bar{P}}$ the subvariety consisting of $L \subset \mathbb{X}_{\bar{P}} \cap W$ such that there is no $(m+1)$-dimensional affine subspace $M \subset \mathbb{X}_{\bar{P}}, M \not \subset W$ containing $L$. 
Theorem 2.11. For any $\bar{d}$, any $m, t \geq 1$, there exists an effective bound $\tilde{r}(m, t, \bar{d})$ with the following property. For any finite field $k=\mathbb{F}_{q}$, any $k$-vector space $V$ and any family $\bar{P} \in \mathcal{P}_{\bar{d}}(V)$ of nc-rank $\geq \tilde{r}(m, t, \bar{d})$, we have that $\frac{\left|Y_{\bar{P}}\right|}{\left|Z_{\bar{P}}\right|} \leq q^{-t}$.

Remark 2.12. The condition that $r(P) \gg 1$ does not imply that $Y_{\bar{P}}=\emptyset$. To see that $r(P) \gg 1$ does not imply the emptiness of the set $Y_{\bar{P}}$ consider the case $V=k^{n}, W=\left\{w \in V: w_{n}=0\right\}, m=1, P=\sum_{i=1}^{n-1} x_{i}^{d}+x_{n}$ and $L=k e_{1}$.

2.1. Applications. In this subsection we provide precise formulations of Theorems 1.4, 1.10 and 1.12.

2.1.1. The surjectivity over algebraically closed fields. We start with a formalization of Theorem 1.4.

Theorem 2.13 (Effective Stillman conjecture). There exists an effective bound $r(m, t, \bar{d})$ with the following property.

For any sequence $\bar{d}$ and $m, t \geq 1$, any algebraically closed field $k$, any $k$-vector space $V$ and any family $\bar{P} \in \mathcal{P}_{\bar{d}}(V)$ of nc-rank $\geq r(m, t, \bar{d})$ the following holds

(1) The map $\kappa_{\bar{P}}$ is surjective.

(2) All fibers of the morphism $\tilde{\kappa}_{\bar{P}}$ are of the same dimension.

(3) The morphism $\tilde{\kappa}_{\bar{P}}$ is flat.

(4) $\bar{P} \subset k\left[V^{\vee}\right]$ is a regular sequence.

\section{Remark 2.14.}

(1) Parts (1) and (2) follow from Theorem 2.9.

(2) The part (3) follows from the parts (1),(2) and Theorem 23.1 in [22]. The part (4) is an immediate consequence of the part (3).

(3) As shown in [1] the part (4) of the theorem implies the validity of an effective Stillman conjecture. 2.10 .

In the formulation of the next result we use notation introduced in Definition

Theorem 2.15. For any $\bar{d}, m, t \geq 1$, there exists effective bound $\tilde{r}(m, t, \bar{d})$ with the following property. For any algebraically closed field $k$, a $k$-vector space $V$ and a family $\bar{P} \in \mathcal{P}_{\bar{d}}(V)$ of nc-rank $\geq \tilde{r}(m, t, \bar{d})$, we have that $\operatorname{dim}\left(\mathbb{Z}_{\bar{P}}\right)-\operatorname{dim}\left(\mathbb{Y}_{\bar{P}}\right) \geq t$.

\subsubsection{Extending weakly polynomial functions.}

\section{Definition 2.16.}

(1) Let $V$ be a $k$-vector space and $X \subset V$. We say that a function $f: X \rightarrow k$ is weakly polynomial of degree $\leq a$ if the restriction $f_{\mid L}$ to any affine subspace $L \subset X$ is a polynomial of degree $\leq a$.

(2) $X$ satisfies $\star^{a}$ if any weakly polynomial function of degree $\leq a$ on $X$ is a restriction of a polynomial function of degree $\leq a$ on $V$.

Below is a formalization of Theorem 1.10 from the introduction. 
Theorem 2.17. For any $\bar{d}$ and $a \geq 1$ there exists an effective bound $r(\bar{d}, a)$ such for any field $k$ which is either finite with $|k|>$ ad or algebraically closed, a $k$ vector space $V$ and a family $\bar{P} \in \mathcal{P}_{\bar{d}}(V)$ of nc-rank $\geq r(\bar{d}, a)$ the subset $X_{\bar{P}} \subset V$ has the property $\star_{a}$.

2.1.3. Nullstellensatz over $\mathbb{F}_{q}$. Below is a formalization of Theorem 1.12 from the introduction.

Theorem 2.18. For any sequence $\bar{d}$, there exists an effective bound $r(\bar{d})>0$ such that the following holds. Let $k=\mathbb{F}_{q}$ be a finite field and let $V$ be a $k$-vector space and $\bar{P}=\left\{P_{i}\right\}$ be a family of $k$-polynomials of degrees $\leq d_{i}$ on $V$ of nc-rank greater than $r(\bar{d})$. Then any polynomial $R$ of degree $\left\langle q / \tilde{d}, \tilde{d}:=\prod_{i=1}^{c} d_{i}\right.$, such that $R(x)=0$ for each $x \in \mathbb{X}_{\bar{P}}(k)$, belongs to the ideal $J(\bar{P}):=\left(P_{1}, \ldots, P_{c}\right)$.

2.2. Acknowledgement. We thank U. Hrushovski for his help with simplifying the proof of Theorem 2.18 .

\section{AnAlysis}

In the main part of this section we prove Theorems 2.9 and 2.11 using the results on equidistribution of high rank families of polynomials. These are based on the technique of the additive combinatorics. At the end of the section we apply these results to prove Theorems 2.13, 2.15 and 1.9.

3.1. Equidistribution of high rank families of polynomials. The most basic result is the following proposition on equidistribution of high rank families of polynomials:

Proposition 3.1. For any sequence $\bar{d}=\left(d_{1}, \ldots, d_{c}\right)$ and any $s \geq 1$ there exists an effective bound $r(\bar{d}, s)$ with the following property. For any finite field $k=\mathbb{F}_{q}$, a $k$ vector space $V$ and a family $\bar{P} \in \mathcal{P}_{\bar{d}}(V)$ of nc-rank $\geq r(\bar{d}, s)$ the map $\bar{P}: V \rightarrow k^{c}$ is $q^{-s}$-uniform.

The main ingredient of this proof comes from the relation between the bias of exponential sums and algebraic rank.

Let $k$ be a finite field, $\operatorname{char}(k)=p,|k|=q$. Let $V$ be a vector space over $k$. We denote $e_{q}(x)=e^{2 \pi i \psi(x) / p}$, where $\psi: k \rightarrow \mathbb{F}_{p}$ is the trace function. Then $e_{q}$ is a non trivial additive character. Let $P: V \rightarrow k$ be a polynomial of degree d. Recall that $\tilde{P}\left(h_{1}, \ldots, h_{d}\right)==\Delta_{h_{d}} \ldots \Delta_{h_{1}} P(x)$ is the multilinear form on $V^{d}$ associated with $P$, and we can write

$$
\tilde{P}\left(h_{1}, \ldots, h_{d}\right)=\sum_{\omega \in\{0,1\}^{d}}(-1)^{|\omega|} P(x+\omega \cdot \bar{h}) ; \quad|\omega|=\sum_{i=1}^{d} \omega_{i}, \quad \omega \cdot \bar{h}=\sum_{i=1}^{d} \omega_{i} h_{i} .
$$

We denote by $\mathbb{E}_{x \in S} f(x)$ the average $|S|^{-1} \sum_{x \in S} f(x)$. 
Definition 3.2 (Gowers norms [6]). For a function $g: V \rightarrow \mathbb{C}$ we define the norm $\|g\|_{U_{d}}$ by

$$
\|g\|_{U_{d}}^{2^{d}}=\mathbb{E}_{x, v_{1}, \ldots v_{d} \in V} \prod_{\omega \in\{0,1\}^{d}} g^{\omega}(x+\omega \cdot \bar{v}),
$$

where $g^{\omega}=g$ if $|\omega|$ is even and $g^{\omega}=\bar{g}$ otherwise.

Definition 3.3 (Analytic rank). The analytic rank of a polynomial $P: V \rightarrow k$ of degree $d$ is defined by $\operatorname{arank}(P)=-\log _{q}\left\|e_{q}(P)\right\|_{U_{d}}$.

The key analytic tool in this paper is the following theorem relating bias and rank.

Theorem 3.4 (Bias-rank).

(1) Let $s, d>0$. There exists $r=r(s, d)$ such that for any finite field $k$ of size $q$, any vector space $V$ over $k$, any polynomial $P: V \rightarrow k$ of degree $d$ the following holds. If $P$ is of nc-rank $>r$ then

$$
\left\|e_{q}(P)\right\|_{U_{d}}^{2^{d}}=\left|\mathbb{E}_{v \in V} e_{q}\left(\tilde{P}\left(h_{1}, \ldots, h_{d}\right)\right)\right|<q^{-s} .
$$

(2) Let $r, d>0$. For any finite field $k$ of size $q$, any vector space $V$ over $k$, any polynomial $P: V \rightarrow k$ of degree $d$, if

$$
\mid \mathbb{E}_{h_{1}, \ldots, h_{d}} e_{q}\left(\tilde{P}\left(h_{1}, \ldots, h_{d}\right) \mid<q^{-r}\right.
$$

for some polynomial, then $P$ is of partition rank $>r$.

Proof. Part (1) was proved for partition rank in increasing generality in [8, 14, 4]. The most general version of the first part can be found at the survey [9] (Theorem 8.0.1). Part (2) for was observed in [19], [23]. Now the Theorem follows from Lemma 2.5.

Remark 3.5. The dependence of $r$ on $s$ in (1) is polynomial, and double exponential in $d$. This was shown for $d=2,3$ in [10, for $d=4$ in 21 and in full generality in [26] (A similar bound but with a weak dependence in $|k|$ was proved independently in [12]).

Remark 3.6. By repeated applications of Cauchy-Schwartz we have that

$$
\left|\mathbb{E}_{x \in V} e_{q}(P(x))\right| \leq\left\|e_{q}(P)\right\|_{U_{d}} .
$$

\section{Remark 3.7.}

(1) We use Claim 1.2 in our proof of Theorems 2.9 and 2.11

(2) Let $P: V \rightarrow k$ be a polynomial of degree $d$ and rank $R$, and let $W \subset V$ be a subspace of codimension $s$. Then the rank of $P_{\mid W}$ is $\geq R-s$.

Proof. We may assume that $\mathbb{V}=\mathbb{A}^{n}$ and $\mathbb{W} \subset \mathbb{V}$ consists of vectors $\left(x_{i}\right), 1 \leq i \leq n$ such that $x_{i}=0$ for $i \leq s$. If the rank of $P_{\mid W}$ is $<R-s$ we can find polynomials $Q_{j}, R_{j}$, with $j \leq r$, fro some $r<R-s$ of degrees $<d$ such that $S_{\mid W} \equiv 0$ where $S:=P-\sum_{j=1}^{r} Q_{j} R_{j}$. Since $S_{\mid W} \equiv 0$ there exists polynomials $T_{i}, 1 \leq i \leq s$ of degrees $<d$ such that $S=\sum_{i=1}^{s} x_{i} T_{i}$. 
Now we can prove Proposition 3.1.

Proof. The number of points on $X_{\bar{P}}^{\bar{b}}=\{x: \bar{P}(x)=\bar{b}\}$ is given by

$$
q^{-c} \sum_{\bar{a} \in k^{c}} \sum_{x \in V} e_{q}\left(\sum_{i=1}^{c} a_{i}\left(P_{i}(x)-b_{i}\right)\right) .
$$

By Theorem 3.4 and Remark 3.6, for any $s>0$ we can choose $r$ so that for any $\bar{a} \neq 0$ we have

$$
\left|\sum_{x \in V} e_{q}\left(\sum_{i=1}^{c} a_{i}\left(P_{i}(x)-b_{i}\right)\right)\right|<q^{-s}|V| .
$$

3.2. Proof of Theorem 2.9. Let $\epsilon>0$ we say that a property holds for $\epsilon$ a.e.s $\in S$ if it holds for all but $(1-\epsilon)|S|$ of the elements in $S$.

Theorem 3.8. For any $\bar{d}$ there exists $C=C(\bar{d})$ such that for any $s, m>0$ there exists an effective bound $r=r(s, \bar{d})$ such that if $\bar{P}=\left(P_{i}\right)_{1 \leq i \leq c}$ is a collection of polynomials on $V=k^{n}$ with $\operatorname{deg} P_{i}=d_{i}$ and the nc-rank $\bar{P}$ is $>r$ then:

(1) For any collection of polynomials $\bar{R}=\left(R_{i}\right)_{1 \leq i \leq c}$, with $R_{i}: k^{m} \rightarrow k$ of degree $d_{i}$, there exist an affine map $w: k^{m} \rightarrow k^{n}$ such that $\bar{P}(w(x))=$ $\bar{R}(x)$. Furthermore, if we denote by $n_{\bar{R}}$ the number of such affine maps, then for any $\bar{R}_{1}, \bar{R}_{2}$ as above $\left|1-n_{\bar{R}_{1}} / n_{\bar{R}_{2}}\right|<q^{-s+m^{C}}$.

(2) If $\bar{P}$ is homogeneous, then for any homogeneous collection $\bar{R}=\left(R_{i}\right)_{1 \leq i \leq c}$, $R_{i}: k^{m} \rightarrow k$ of degree $d_{i}$, there exist a linear map $w: k^{m} \rightarrow k^{n}$ such that $\bar{P}(w(x))=\bar{R}(x)$. Furthermore, if we denote by $n_{\bar{R}}$ the number of such linear maps, then for any $\bar{R}_{1}, \bar{R}_{2}$ as above $\left|1-n_{\bar{R}_{1}} / n_{\bar{R}_{2}}\right|<q^{-s+m^{C}}$.

Proof. We provide two proofs of the Theorem: one which is valid only in the case where $\operatorname{char}(k)>d$, but is more algebraic in nature, and the other relies on multiple applications of Cauchy-Schwartz.

We start with the proof in the case where $\operatorname{char}(k)>d$. Since the proof for general $d$ involves many indices, we first prove the case when $c=1$ and $d=2$ so as to make the argument clear.

We are given $P(t)=\sum_{1 \leq i \leq j \leq n} a_{i j} t_{i} t_{j}+\sum_{1 \leq i \leq n} a_{i} t_{i}+a$ of rank $r$. Note that for any linear form $l(t)=\sum_{i=1}^{n} c_{i} t_{i}$ we have that $P(t)+l(t)$ is of rank $\geq r$. 
Denote $w(x)=\left(w^{1}(x)+s^{1}, \ldots, w^{n}(x)+s^{n}\right)$, where the $w^{i}$ are linear forms. We can write

$$
\begin{aligned}
P(w(x)) & =\sum_{1 \leq i \leq j \leq n} a_{i j}\left(w^{i}(x)+s^{i}\right)\left(w^{j}(x)+s^{j}\right)+\sum_{1 \leq i \leq n} a_{i}\left(w^{i}(x)+s^{i}\right)+a \\
& =\sum_{1 \leq i \leq j \leq n} a_{i j} \sum_{k, l=1}^{m} w_{k}^{i} w_{l}^{j} x_{k} x_{l}+\sum_{1 \leq i \leq n} a_{i} \sum_{k=1}^{m} w_{k}^{i} x_{k} \\
& +\sum_{1 \leq i \leq j \leq n} \sum_{k=1}^{m} a_{i j}\left(s^{i} w_{k}^{j}+s^{j} w_{k}^{i}\right) x_{k}+\sum_{1 \leq i \leq j \leq n} a_{i j} s^{i} s^{j}+\sum_{1 \leq i \leq n} a_{i} s^{i}+a,
\end{aligned}
$$

which we can write as

$$
\begin{aligned}
& \sum_{1 \leq k<l \leq m} \sum_{1 \leq i \leq j \leq n} a_{i j}\left(w_{k}^{i} w_{l}^{j}+w_{l}^{i} w_{k}^{j}\right) x_{k} x_{l}+\sum_{1 \leq l \leq m} \sum_{1 \leq i \leq j \leq n} a_{i j} w_{l}^{i} w_{l}^{j} x_{l}^{2} \\
& +\sum_{k=1}^{m}\left[\sum_{1 \leq i \leq n} a_{i} w_{k}^{i}+\sum_{1 \leq i \leq j \leq n} a_{i j}\left(s^{i} w_{k}^{j}+s^{j} w_{k}^{i}\right)\right] x_{k}+\sum_{1 \leq i \leq j \leq n} a_{i j} s^{i} s^{j}+\sum_{1 \leq i \leq n} a_{i} s^{i}+a .
\end{aligned}
$$

Our aim is to show that the collection of coefficients for all monomials in the variables $x_{j}, 1 \leq j \leq m$, is of rank $\geq r$ (as polynomials in $\left.w^{i}, s^{i}\right)$ :

Claim 3.9. The collection below is of rank $\geq r-1$.

$$
\begin{aligned}
& \left\{\sum_{1 \leq i \leq j \leq n} a_{i j}\left(w_{k}^{i} w_{l}^{j}+w_{l}^{i} w_{k}^{j}\right)\right\}_{1 \leq k<l \leq m} \bigcup\left\{\sum_{1 \leq i \leq j \leq n} a_{i j}\left(s^{i} w_{k}^{j}+s^{j} w_{k}^{i}\right)+\sum_{1 \leq i \leq n} a_{i} w_{k}^{i}\right\}_{1 \leq k \leq m} \\
& \bigcup\left\{\sum_{1 \leq i \leq j \leq n} a_{i j} s^{i} s^{j}+\sum_{1 \leq i \leq n} a_{i} s^{i}\right\} \bigcup\left\{\sum_{1 \leq i \leq j \leq n} a_{i j} w_{l}^{i} w_{l}^{j}\right\}_{1 \leq l \leq m}
\end{aligned}
$$

Proof. We need to show that any non-trivial linear combination

$$
\begin{aligned}
& \sum_{1 \leq k<l \leq m} b_{k l}\left[\sum_{1 \leq i \leq j \leq n} a_{i j}\left(w_{k}^{i} w_{l}^{j}+w_{l}^{i} w_{k}^{j}\right)\right]+\sum_{1 \leq l \leq m} b_{l l}\left[\sum_{1 \leq i \leq j \leq n} a_{i j} w_{l}^{i} w_{l}^{j}\right] \\
& +\sum_{1 \leq k \leq m} c_{k}\left[\sum_{1 \leq i \leq j \leq n} a_{i j}\left(s^{i} w_{k}^{j}+s^{j} w_{k}^{i}\right)+\sum_{1 \leq i \leq n} a_{i} w_{k}^{i}\right]+d\left[\sum_{1 \leq i \leq j \leq n} a_{i j} s^{i} s^{j}+\sum_{1 \leq i \leq n} a_{i} s^{i}\right]
\end{aligned}
$$

is of rank $\geq r$. Suppose $b_{11} \neq 0$. Then we can write the above as

$$
b_{11} P\left(w_{1}\right)+l_{w_{2}, \ldots, w_{m}, s}\left(w_{1}\right)
$$

where $w_{j}=\left(w_{j}^{1}, \ldots, w_{j}^{n}\right)$, and $l_{w_{2}, \ldots, w_{m}, s}$ is affine in $w_{1}$, so as a polynomial in $w_{1}$ this is of rank $\geq r$ and thus also of rank $\geq r$ as a polynomial in $w$. Similarly in the case where $b_{l l} \neq 0$, for some $1 \leq l \leq m$.

Suppose $b_{12} \neq 0$. We can write the above as

$$
(*) \quad b_{12} Q\left(w_{1}, w_{2}\right)+l_{w_{3}, \ldots, w_{m}, s}^{1}\left(w_{1}\right)+l_{w_{3}, \ldots, w_{m}, s}^{2}\left(w_{2}\right)
$$


where $Q: V^{2} \rightarrow k$, and $Q(t, t)=2 P(t)$, and $l_{w_{3}, \ldots, w_{m}, s}^{i}: V \rightarrow k$ are affine maps. Thus restricted to the subspace $W$ where $w_{1}=w_{2}$ we get that $(*)$ is of rank $\geq r$ and thus of rank $\geq r-1$ on $W$. Similarly if $b_{k l} \neq 0$ for some $k<l$. A similar analysis for the cases when $c_{k}$ or $d_{k}$ are not zero yields the desired result.

If $P$ is homogeneous $P(t)=\sum_{1 \leq i \leq j \leq n} a_{i j} t_{i} t_{j}$ of rank $r$, and $w: k^{m} \rightarrow k^{n}$ a linear map, we can write

$$
P(w(x))=\sum_{1 \leq i \leq j \leq n} a_{i j} w^{i}(x) w^{j}(x)=\sum_{1 \leq i \leq j \leq n} a_{i j} \sum_{k, l=1}^{m} w_{k}^{i} w_{l}^{j} x_{k} x_{l}
$$

which we can write as

$$
\sum_{1 \leq k<l \leq m} \sum_{1 \leq i \leq j \leq n} a_{i j}\left(w_{k}^{i} w_{l}^{j}+w_{l}^{i} w_{k}^{j}\right) x_{k} x_{l}+\sum_{1 \leq l \leq m} \sum_{1 \leq i \leq j \leq n} a_{i j} w_{l}^{i} w_{l}^{j} x_{l}^{2}
$$

By Claim 3.9 the collection of coefficients for all monomials in the variables $x_{j}, 1 \leq j \leq m$, is also of rank $\geq r-1$.

For $d>2$ we perform a similar computation. To simplify the notation we carry it out in the case $P, R$ are homogeneous; the non-homogeneous case is similar. In this case it suffices to use linear maps. Denote $\mathcal{I}_{n}=\left\{I=\left(i_{1}, \ldots, i_{d}\right): 1 \leq\right.$ $\left.i_{1} \leq \ldots \leq i_{d} \leq n\right\}$, and for $t \in k^{n}$ denote by $t_{I}=\prod_{j=1}^{d} t_{i_{j}}$. Let $P$ be a degree $d$ polynomial $P(t)=\sum_{I \in \mathcal{I}_{n}} a_{I} t_{I}$ on $k^{n}$ of rank $r$.

Let $w: k^{m} \rightarrow k^{n}$ be a linear map. We can write

$$
P(w(x))=\sum_{I \in \mathcal{I}_{n}} a_{I}\left(\prod_{j=1}^{d} w^{i_{j}}(x)\right)=\sum_{I \in \mathcal{I}_{n}} a_{I} \sum_{l_{1}, \ldots, l_{d}=1}^{m}\left(\prod_{j=1}^{d} w_{l_{j}}^{i_{j}}\right) x_{l_{1}} \ldots x_{l_{d}} .
$$

We rewrite this as

$$
\sum_{l_{1}, \ldots, l_{d}=1}^{m} \sum_{I \in \mathcal{I}_{n}} a_{I}\left(\prod_{j=1}^{d} w_{l_{j}}^{i_{j}}\right) x_{l_{1}} \ldots x_{l_{d}}=\sum_{l \in \mathcal{I}_{m}}\left(\sum_{I \in \mathcal{I}_{n}} a_{I} \sum_{\sigma \in S(l)}\left(\prod_{j=1}^{d} w_{\sigma_{j}}^{i_{j}}\right)\right) x_{l_{1}} \ldots x_{l_{d}}
$$

where $S(l)$ is the set of permutations of $l_{1}, \ldots l_{d}$.

Once again, our aim is to show that the collection of coefficients for all possible monomials in the variables $x_{j}, 1 \leq j \leq m$, is of rank $\geq r$ :

Claim 3.10. The collection below is of rank $\geq r-d+1$ :

$$
\left\{\sum_{I \in \mathcal{I}_{n}} a_{I} \sum_{\sigma \in S(l)} \prod_{j=1}^{d} w_{\sigma_{j}}^{i_{j}}\right\}_{l \in \mathcal{I}_{m}}
$$


Proof. Consider a linear combination of the above collection with coefficient $c_{l} \neq 0$ for some $l \in \mathcal{I}_{m}$. Consider

$$
Q\left(w_{l_{1}}, \ldots, w_{l_{d}}\right)=\sum_{I \in \mathcal{I}_{n}} a_{I} \sum_{\sigma \in S(l)} \prod_{j=1}^{d} w_{\sigma_{j}}^{i_{j}} .
$$

Then if $\Delta_{w} P(y)=P(y+w)-P(y)$ then $Q\left(w_{l_{1}}, \ldots, w_{l_{d}}\right)=\Delta_{w_{l_{1}}} \ldots \Delta_{w_{l_{d}}} P(y)$. Now we can write the given linear combination as $c_{l} Q\left(w_{l_{1}}, \ldots, w_{l_{d}}\right)+T\left(w_{l_{1}}, \ldots, w_{l_{d}}\right)$ where $T$ is a function of lower degree in $w_{l_{1}}, \ldots, w_{l_{d}}$ (it also depends on the $w_{t}$ for $t \neq l_{i}$, for $\left.1 \leq i \leq d\right)$. Since $(\operatorname{char}(k), d)=1$, by Remark $3.7(2)$ we have that the rank of $Q$ is the same as that of $P$, and thus the rank of the collection is the same as rank of $P$.

When $c>1$,we are given $P_{s}(t)=\sum_{I \in \mathcal{I}_{s}} a_{I}^{s} t_{I}, 1 \leq s \leq c$, of rank $r$, where $\mathcal{I}_{d_{s}}(n)$ is the set of ordered tuples $I=\left(i_{1}, \ldots, i_{d_{s}}\right)$ with $1 \leq i_{1} \leq \ldots \leq i_{d_{s}} \leq n$, and $t_{I}=t_{i_{1}} \ldots t_{i_{s}}$.

Note that for any polynomials $l_{s}(t)$ of degrees $<d_{s}$ we have that $\left\{P_{s}(t)+l_{s}(t)\right\}$ is also of rank $>r$.

We can write

$$
P_{s}(w(x))=\sum_{I \in \mathcal{I}_{d_{s}}(n)} a_{I}^{s} w^{I}(x)=\sum_{I \in \mathcal{I}_{d_{s}}(n)} a_{I}^{s} \sum_{l_{1}, \ldots, l_{d_{s}}=1}^{m} w_{l_{1}}^{i_{1}} \ldots w_{l_{d_{s}}}^{i_{d_{s}}} x_{l_{1}} \ldots x_{l_{d_{s}}},
$$

where $w^{I}=\prod_{i \in I} w^{i}$. For $\left(l_{1}, \ldots, l_{d_{s}}\right) \in \mathcal{I}_{d_{s}}(m)$ the term $x_{l_{1}} \ldots x_{l_{d_{s}}}$ has as coefficient

$$
\sum_{\sigma \in S_{d_{s}}} \sum_{I \in \mathcal{I}_{s}(n)} a_{I}^{s} w_{l_{\sigma(1)}}^{i_{1}} \ldots w_{l_{\sigma\left(d_{s}\right)}}^{i_{d_{s}}}
$$

We wish to show that the collection

$$
\left\{\sum_{\sigma \in S_{d_{s}}} \sum_{I \in \mathcal{I}_{d_{s}}(n)} a_{I}^{s} w_{l_{\sigma(1)}}^{i_{1}} \ldots w_{l_{\sigma\left(d_{s}\right)}}^{i_{d_{s}}}\right\}_{1 \leq s \leq c,\left(l_{1}, \ldots, l_{d_{s}}\right) \in \mathcal{I}_{s}(m)}
$$

is of rank $>r$. Write $[1, c]=\bigcup_{f=2}^{d} C_{f}$ where $C_{f}=\left\{s: d_{s}=f\right\}$.

We need to show that for any $f=2, \ldots, d$ if $B=\left(b_{\left(l_{1}, \ldots, l_{d_{s}}\right)}^{s}\right)_{s \in C_{f},\left(l_{1}, \ldots, l_{d_{s}}\right) \in \mathcal{I}_{d_{s}}(m)}$ is not $\overline{0}$, then

$$
\sum_{s \in C_{f}} \sum_{\left(l_{1}, \ldots, l_{f}\right)} b_{\left(l_{1}, \ldots, l_{f}\right)}^{s} \sum_{\sigma \in S_{f}} \sum_{I \in \mathcal{I}_{f}(n)} a_{I}^{s} w_{l_{\sigma(1)}}^{i_{1}} \ldots w_{l_{\sigma(f)}}^{i_{f}}
$$

is of rank $>r$. Suppose $\left(b_{\left(l_{1}, \ldots, l_{f}\right)}^{s}\right)_{s \in C_{f}} \neq \overline{0}$. Then restricted to the subspace $w_{l_{1}}=\ldots=w_{l_{f}}$ we can write the above as

$$
\sum_{s \in C_{f}} b_{\left(l_{1}, \ldots, l_{f}\right)}^{s}(f !) P_{s}\left(w_{l_{1}}\right)+R(w)
$$


where $w_{j}=\left(w_{j}^{1}, \ldots, w_{j}^{n}\right)$, and $R(w)$ is of lower degree in $w_{l_{1}}$, so as a polynomial in $w_{l_{1}}$ this is of rank $>r$ and thus also of rank $>r$ as a polynomial in $w$.

Now (1), (2) follow from Proposition [3.1, since $\left|\mathcal{I}_{m}\right|=m^{C(\bar{d})}$.

We give an alternative proof that is valid for all characteristic. Following the computations above it suffices to show the following Claim:

Claim 3.11. For any $t>0$ there exists $r=r(t, \bar{d})$ such that if the nc-rank of $\bar{P}$ is $>r$ then for any polynomial $Q$ of degree $2 \leq f \leq c$ that is a non trivial combination of the polynomials in the collection

$$
\left\{\sum_{\sigma \in S_{f}} \sum_{I \in \mathcal{I}_{f}(n)} a_{I}^{s} w_{l_{\sigma(1)}}^{i_{1}} \ldots w_{l_{\sigma(f)}}^{i_{f}}\right\}_{s \in C_{f},\left(l_{1}, \ldots, l_{f}\right) \in \mathcal{I}_{f}(m)}
$$

we have that $\left|\mathbb{E} e_{q}(Q)\right|<q^{-t}$.

Proof. Let $Q$ be of degree $f$, and write

$$
Q=\sum_{\left(l_{1}, \ldots, l_{f}\right)} \sum_{s \in C_{f}} b_{\left(l_{1}, \ldots, l_{f}\right)}^{s} \sum_{\sigma \in S_{f}} \sum_{I \in \mathcal{I}_{f}(n)} a_{I}^{s} w_{l_{\sigma(1)}}^{i_{1}} \ldots w_{l_{\sigma(f)}}^{i_{f}}
$$

where $\bar{b}_{\left(l_{1}, \ldots, l_{f}\right)}=\left(b_{\left(l_{1}, \ldots, l_{f}\right)}^{s}\right)_{s \in C_{f}} \neq 0$ for some $\left(l_{1}, \ldots, l_{f}\right) \in \mathcal{I}_{f}(m)$. Observe that any $\left(l_{1}, \ldots, l_{f}\right)$ determines a unique set of variables $w_{l_{1}}, \ldots, w_{l_{f}}$, thus after $f$ applications of the Cauchy-Schwarz inequality we can isolate this collection and arrive at the multilinear from associated with differentiating

$$
\sum_{s \in C_{f}} b_{\left(l_{1}, \ldots, l_{f}\right)}^{s} \sum_{\sigma \in S_{f}} \sum_{I \in \mathcal{I}_{f}(n)} a_{I}^{s} w_{l_{\sigma(1)}}^{i_{1}} \ldots w_{l_{\sigma(f)}}^{i_{f}}
$$

with respect to the associated set of variables. Namely we have

$$
\left|e_{q}(Q)\right|_{U_{1}} \leq\left\|e_{q}\left(\sum_{s \in C_{f}} b_{\left(l_{1}, \ldots, l_{f}\right)}^{s} \sum_{\sigma \in S_{f}} \sum_{I \in \mathcal{I}_{f}(n)} a_{I}^{s} w_{l_{\sigma(1)}}^{i_{1}} \ldots w_{l_{\sigma(f)}}^{i_{f}}\right)\right\|_{U_{f}}
$$

But the latter is equal to

$$
\mathbb{E}_{w_{l_{1}}, \ldots, w_{l_{f}}} e_{q}\left(\Delta_{w_{l_{1}}} \ldots \Delta_{w_{l_{d}}} \bar{b}_{\left(l_{1}, \ldots, l_{f}\right)} \cdot \bar{P}^{f}(x)\right) .
$$

where $\bar{P}^{f}=\left(P_{s}\right)_{s \in C_{f}}$. Now by Theorem 3.4 we can choose $r$ such that if $\bar{P}$ is of rank $>r$ then the above is $<q^{-s}$.

This completes the proof of Theorem 3.8 . 
3.3. Proof of Theorem 2.11. In this subsection we prove Theorem 2.11.

Let $V$ be a vector space and $l: V \rightarrow k$ be a non-constant linear function. For any subset $I$ of $k$ we denote $\mathbb{W}_{I}=\{v \in \mathbb{V} \mid l(v) \in I\}$ so that $\mathbb{W}_{b}=\mathbb{W}_{\{b\}}$, for $b \in k$. For $\mathbb{X} \subset \mathbb{V}$ we write $\mathbb{X}_{I}=\mathbb{X} \cap \mathbb{W}_{I}$.

Theorem 2.11 follows from the following proposition:

Proposition 3.12. Fix $\bar{d}=\left\{d_{i}\right\}$ and $m, s>0$. Let $d:=\max _{i} d_{i}$. There exists an effective bound $r=r(\bar{d}, s, m)$ such that for any finite field $k$, any $k$-vector space $\mathbb{V}$ and $\bar{P} \in \mathcal{P}_{\bar{d}}(\mathbb{V})$ of nc-rank $>r$ the following holds. For any $b \in k$ and $q^{-s}$-almost any affine $m$-dimensional subspace $L \subset X_{b}$ there exists an $(m+1)$-dimensional affine subspace $M \subset X$ containing $L$ such that $M \cap X_{0} \neq \emptyset$.

Proof. We fix $d$ and define $d^{\prime}=\min (d+1, q)$. Let $M_{0}=\left\{a_{0}, \ldots, a_{d}\right\} \subset k$ be a subset of $d^{\prime}$ distinct points. To simplify notations we assume that $a_{0}=0$.

Claim 3.13. Let $Q(x)$ be a polynomial of degree $\leq d$ such that $Q_{\mid M_{0}} \equiv 0$. Then $Q(a)=0$ for all $a \in k$.

Proof. Since any polynomial of degree $\leq d$ in one variable vanishing at $d+1$ point is equal to 0 , the claim is true if $q \geq d+1$. On the other hand if $d \geq q$ then there is nothing to prove.

Let $J(d)$ be the subset of $[0, d]^{m+1}$ of tuples $t=\left(t_{1}, \ldots, t_{m+1}\right)$ such that $0 \leq$ $t_{m+1} \leq \ldots \leq t_{1}$. Let $T^{m+1}:=\left\{a_{t}=\left(a_{t_{1}}, \ldots, a_{t_{m+1}}\right): t \in J(d)\right\} \subset k^{m+1}$.

Claim 3.14. Let $Q\left(x_{1}, \ldots, x_{m+1}\right)$ be a polynomial of degree $\leq d$ such that $Q_{\mid T^{m+1}} \equiv 0$. Then $Q=0$.

Proof. Our proof is by induction in $m$ and for a fixed $m$ by induction in $d$.

Consider first the case when $m=1$. We will write $x, y$ instead of $x_{1}, x_{2}$. We have $T^{2}=\left\{\left(a_{t_{1}}, a_{t_{2}}\right): 0 \leq t_{2} \leq t_{1} \leq d\right\}$.

We prove the claim by induction in $d$. Let $Q=\sum_{a, b} q_{a, b} x^{a} y^{b}$, with $a+b \leq d$. The restriction of $Q$ to the line $\{y=0\}$ is equal to $Q^{0}(x)=\sum_{a \leq d} q_{a, 0} x^{a}$. Since $Q_{\mid T^{2}}^{0} \equiv 0$ we see that $Q^{0}=0$. So $Q(x, y)=y Q^{\prime}(x, y)$. So, by the inductive assumption, we have $Q^{\prime} \equiv 0$.

Assume now the validity of Claim for polynomials in $m$ variables and for polynomials in $m+1$ variables degree $\leq d-1$. Let $Q\left(x_{1}, \ldots, x_{m+1}\right)$ be a polynomial of degree $\leq d$ such that $Q_{\mid T^{m+1}} \equiv 0$. Let $R$ be the restriction of $Q$ on the subspace of points $\left(x_{1}, \ldots, x_{m+1}\right)$ such that $x_{m+1}=0$. Since $R_{\mid T^{m}} \equiv 0$ it follows from the inductive assumption that $R \equiv 0$ and therefore $Q=Q^{\prime} x_{m+1}$ where $\operatorname{deg}\left(Q^{\prime}\right)=d-1$. Since $Q_{\mid T}^{\prime} \equiv 0$ we see from the inductive assumption that $Q^{\prime} \equiv 0$.

Denote $I(d)$ the set of indexes

$$
I(d)=\left\{t:=\left(t_{1}, \ldots, t_{m+1}\right) \in J(d): 1 \leq t_{m+1}\right\} .
$$


An affine $m$-dimensional subspace in $X_{b}$ is parametrized as $\left\{x+\sum_{i=1}^{m} s_{i} y_{i} ; s_{i} \in k\right\}$, such that for all $2 \leq e \leq d$, all $P_{j}^{e} \in \bar{P}^{e}$ we have

$$
\text { (*) } \quad P_{j}^{e}\left(x+\sum_{i=1}^{m} s_{i} y_{i}\right)=0, l(x)=b, l\left(y_{i}\right)=0 .
$$

Let $Y$ be the set of $(x, \bar{y})$ satisfying $(*)$.

We need to show that almost every $(x, \bar{y}) \in Y$ we can find $z$ such that for all $2 \leq e \leq d$, all $P_{j}^{e} \in \bar{P}^{e}$ we have

$$
\forall s, s_{1}, \ldots, s_{m} \in k, \quad P_{j}^{e}\left(x+\sum_{i=1}^{m} s_{i} y_{i}+s z\right)=0, l(z)=-b,
$$

or alternatively

$\forall s, s_{1}, \ldots, s_{m} \in k, \quad P_{j}^{e}\left(x+\sum_{i=1}^{m} s_{i} y_{i}+s z\right)=0, l\left(x+\sum_{i=1}^{m} s_{i} y_{i}+s z\right)=(1-s) b$.

By Claim [3.14, since $P_{j}^{e}$ is of degree $e$, we can reduce this system to

$$
P_{j}^{e}\left(x+\sum_{i=1}^{m} a_{t_{i}} y_{i}+a_{m+1} z\right)=0, l\left(x+\sum_{i=1}^{m} a_{t_{i}} y_{i}+a_{t_{m+1}} z\right)=\left(1-a_{t_{m+1}}\right) b, \quad t \in I(e) .
$$

Denote $I=\sum_{e \in[d]}|I(e)|$. Fix $(x, \bar{y}) \in Y$ and estimate the number of solutions $A(x, \bar{y})$ to the above system of equations, which is given by

$$
\begin{aligned}
& q_{z}^{-2 I} \sum_{e \in[d], \bar{c}_{t}^{e}: t^{e} \in I(e), h_{t}: t \in I(d)} \\
& e_{q}\left(\sum_{e \in[d]} \sum_{t^{e}} \bar{c}_{t^{e}}^{e} \cdot \bar{P}^{e}\left(x+\sum_{i=1}^{m} a_{t_{i}^{e}} y_{i}+a_{t_{m+1}^{e}} z\right)+\sum_{t} h_{t}\left(l\left(x+\sum_{i=1}^{m} a_{t_{i}} y_{i}+a_{t_{m+1}} z\right)+\left(a_{t_{m+1}}-1\right) b\right)\right) .
\end{aligned}
$$

Suppose $\bar{c}_{t^{e}}^{e}=0$ for all $t^{e}$, but $\bar{h} \neq 0$, and recall that $l(x)=b, l\left(y_{i}\right)=0$. We get

$$
\begin{aligned}
& \sum_{z} e_{q}\left(\sum_{t} h_{t}\left(l\left(x+\sum_{i=1}^{m} a_{t_{i}} y_{i}+a_{t_{m+1}} z\right)+\left(a_{t_{m+1}}-1\right) b\right)\right) \\
& =\sum_{z} e_{q}\left(\sum_{t} h_{t}\left(a_{t_{m+1}} l(z)+a_{t_{m+1}} b\right)\right) .
\end{aligned}
$$

Now if $\sum_{t} h_{t} a_{t_{m+1}} l(z) \not \equiv 0$ then the sum is 0 . Otherwise also $\sum_{t} h_{t} a_{t_{m+1}} b=0$ so that the sum is $|V|$.

Now suppose $\bar{c}_{t_{0}}^{e} \neq 0$ for some $t_{0}$, and let $e$ be the largest degree for which this holds. Let

$$
T(x, y, z)=\sum_{t^{e}} \bar{c}_{t^{e}}^{e} \cdot \bar{P}^{e}\left(x+\sum_{i=1}^{m} a_{t_{i}^{e}} y_{i}+a_{t_{m+1}^{e}} z\right)+Q(x, y, z)
$$


where $Q$ is of degree $<e$. We estimate

$$
B_{t_{0}}=\mathbb{E}_{x, \bar{y} \in V}\left|\mathbb{E}_{z} e_{q}(T(x, y, z))\right|^{2} .
$$

Lemma 3.15. For any functions $f_{t}: V \rightarrow \mathbb{C},\left\|f_{t}\right\|_{\infty} \leq 1, t \in I(d)$, we have $\left|\mathbb{E}_{x, \bar{y}, z, z^{\prime}} \prod_{t \in I(d)} f_{t}\left(x+\sum_{i=1}^{m} a_{t_{i}} y_{i}+a_{t_{m+1}} z\right) \bar{f}_{t}\left(x+\sum_{i=1}^{m} a_{t_{i}} y_{i}+a_{t_{m+1}} z+a_{t_{m+1}} z^{\prime}\right)\right| \leq\left\|f_{t_{0}}\right\|_{U_{d}}$.

Proof. To simplify the notation we prove this in the case $m=1$. Without loss of generality $a_{1}=1$ (make a change of variables $y \rightarrow a_{1}^{-1} y, z \rightarrow a_{1}^{-1} z$ ). We prove this by induction on $d$. When $d=1, I(d)=\{(1,1)\}$, and the claim in this case follow from the following inequality

$\left|\mathbb{E}_{x, y, z, z^{\prime}} f_{1}(x+y+z) f_{2}\left(x+y+z+z^{\prime}\right)\right|=\left|\mathbb{E}_{z, z^{\prime}} f_{1}(z) f_{2}\left(z^{\prime}\right)\right| \leq\left|\mathbb{E}_{z} f_{i}(z)\right| \leq\left\|f_{t_{0}}\right\|_{U_{1}}$.

Assume $d>1$. We can write the average as

$$
\begin{array}{r}
\mathbb{E}_{x, y, z, z^{\prime}} \prod_{(i, j) \in I(d-1)} f_{i, j}\left(x+a_{i} y+a_{j} z\right) \bar{f}_{i, j}\left(x+a_{i} y+a_{j} z+a_{j} z^{\prime}\right) \\
\prod_{1 \leq j \leq d} f_{d, j}\left(x+a_{d} y+a_{j} z\right) \bar{f}_{d, j}\left(x+a_{d} y+a_{j} z+a_{j} z^{\prime}\right) .
\end{array}
$$

Shifting $x$ by $a_{d} y$ we get

$$
\begin{gathered}
\mathbb{E}_{x, y, z, z^{\prime}} \prod_{(i, j) \in I(d-1)} f_{i, j}\left(x+\left(a_{i}-a_{d}\right) y+a_{j} z\right) \bar{f}_{i, j}\left(x+\left(a_{i}-a_{d}\right) y+a_{j} z+a_{j} z^{\prime}\right) \\
\prod_{1 \leq j \leq d} f_{d, j}\left(x+a_{j} z\right) \bar{f}_{d, j}\left(x+a_{j} z+a_{j} z^{\prime}\right) .
\end{gathered}
$$

Applying the Cauchy-Schwarz inequality we can bound the above as

$$
\begin{gathered}
{\left[\mathbb{E}_{x, y, y^{\prime}, z, z^{\prime}} \prod_{(i, j) \in I(d-1)} f_{i, j}\left(x+\left(a_{i}-a_{d}\right) y+a_{j} z\right) \bar{f}_{i, j}\left(x+\left(a_{i}-a_{d}\right) y+a_{j} z+a_{j} z^{\prime}\right)\right.} \\
\prod_{(i, j) \in I(d-1)} \bar{f}_{i, j}\left(x+\left(a_{i}-a_{d}\right) y+\left(a_{i}-a_{d}\right) y^{\prime}+a_{j} z\right) \\
\left.f_{i, j}\left(x+\left(a_{i}-a_{d}\right) y+\left(a_{i}-a_{d}\right) y^{\prime}+a_{j} z+a_{j} z^{\prime}\right)\right]^{1 / 2} .
\end{gathered}
$$

Shifting $x$ by $a_{d} y$ and rearranging we get

$$
\begin{aligned}
& {\left[\mathbb{E}_{x, y, y^{\prime}, z, z^{\prime}} \prod_{(i, j) \in I(d-1)} f_{i, j}\left(x+a_{i} y+a_{j} z\right) \bar{f}_{i, j}\left(x+a_{i} y+\left(a_{i}-a_{d}\right) y^{\prime}+a_{j} z\right)\right.} \\
& \left.\quad \prod_{(i, j) \in I(d-1)} \bar{f}_{i, j}\left(x+a_{i} y+a_{j} z+a_{j} z^{\prime}\right) f_{i, j}\left(x+a_{i} y+\left(a_{i}-a_{d}\right) y^{\prime}+a_{j} z+a_{j} z^{\prime}\right)\right]^{1 / 2} .
\end{aligned}
$$

Now if we denote

$$
g_{i, j, y^{\prime}}(x)=f_{i, j}(x) \bar{f}_{i, j}\left(x+\left(a_{i}-a_{d}\right) y^{\prime}\right),
$$


then by the induction hypothesis we get that the above is bounded by

$$
\left[\mathbb{E}_{y^{\prime}}\left\|g_{i, j, y^{\prime}}\right\|_{U_{d-1}}\right]^{1 / 2} \leq\left\|f_{i, j}(x)\right\|_{U_{d}}
$$

for any $(i, j) \in I(d-1)$.

We do a similar computation for $(i . j) \in I(d) \backslash\{I(d-1),(d, 1)\}$, splitting

$$
\begin{gathered}
\mathbb{E}_{x, y, z, z^{\prime}} \prod_{(i, j) \in I(d-1)} f_{i+1, j+1}\left(x+a_{i+1} y+a_{j+1} z\right) \bar{f}_{i+1, j+1}\left(x+a_{i+1} y+a_{j+1} z+a_{j+1} z^{\prime}\right) \\
\prod_{1 \leq j \leq d} f_{j, 1}\left(x+a_{j} y+z\right) \bar{f}_{j, 1}\left(x+a_{j} y+z+z^{\prime}\right),
\end{gathered}
$$

and shifting $x$ by $z$ to get

$$
\begin{aligned}
\mathbb{E}_{x, y, z, z^{\prime}} & \prod_{(i, j) \in I(d-1)} f_{i+1, j+1}\left(x-z+a_{i+1} y+a_{j+1} z\right) \bar{f}_{i+1, j+1}\left(x-z+a_{i+1} y+a_{j+1} z+a_{j+1} z^{\prime}\right) \\
& \prod_{1 \leq j \leq d} f_{j, 1}\left(x+a_{j} y\right) \bar{f}_{j, 1}\left(x+a_{j} y+z^{\prime}\right) .
\end{aligned}
$$

The only term left uncovered is $f_{d, 1}$, so we split

$$
\begin{gathered}
\mathbb{E}_{x, y, z, z^{\prime}} \prod_{(i, j) \in I(d-1)} f_{i+1, j}\left(x+a_{i+1} y+a_{j} z\right) \bar{f}_{i+1, j}\left(x+a_{i+1} y+a_{j} z+a_{j} z^{\prime}\right) \\
\prod_{1 \leq i \leq d} f_{i, i}\left(x+a_{i} y+a_{i} z\right) \bar{f}_{i, i}\left(x+a_{i} y+a_{i} z+a_{i} z^{\prime}\right) .
\end{gathered}
$$

We make the change of variable $z \rightarrow z-y$ to get

$$
\begin{gathered}
\mathbb{E}_{x, y, z, z^{\prime}} \prod_{(i, j) \in I(d-1)} f_{i+1, j}\left(x+a_{i+1} y+a_{j}(z-y)\right) \bar{f}_{i+1, j}\left(x+a_{i+1} y+a_{j}(z-y)+a_{j} z^{\prime}\right) \\
\prod_{1 \leq i \leq d} f_{i, i}\left(x+a_{i} z\right) \bar{f}_{i, i}\left(x+a_{i} z+a_{i} z^{\prime}\right) .
\end{gathered}
$$

Observe that the argument of $f_{d, 1}$ is $\left(x+\left(a_{d}-a_{1}\right) y+a_{1} z\right)$, so that the coefficient of $y$ is not zero. Now proceed as in previous cases.

By the Lemma 3.15 we obtain that $B_{t_{0}}$ is bounded by $\left\|e_{q}\left(\bar{c}_{t_{0}^{e}}^{e} \cdot \bar{P}^{e}\right)\right\|_{U_{e}}$. By Theorem 3.4 there exists an effective bound $r=r(s, d)$, such that if $P$ is of rank $>r$ then $\left\|e_{q}\left(\bar{c}_{t_{0}^{e}}^{e} \cdot \bar{P}^{e}\right)\right\|_{U_{e}}<q^{-s}$. It follows that we can choose $r$ so that for $q^{-s}$-almost all $(x, y) \in Y$, the number of solutions $A(x, \bar{y})$ is bounded below by $|V| q^{-4 I}$. 
3.4. Proof of Theorems 2.13 and 2.15. We fix $m, d$ and $v$. As follows from Theorem 2.9, there exists an effective bound $r=r(m, d, c)$ such that for any finite field $\mathbb{F}_{q}$, any $\mathbb{F}_{q}$-vector space $\mathbb{V}$ the map $\tilde{\kappa}_{P}\left(\mathbb{F}_{q}\right)$ is surjective for any family $\bar{P}=\left(P_{i}\right)$ of polynomials $P_{i} \in \mathcal{P}_{d}(\mathbb{V})$ such that $r_{n c}(\bar{P}) \geq r$.

We now consider the case when $k$ is an algebraically closed field.

3.4.1. The surjectivity of $\tilde{\kappa}_{P}(k)$. We fix $\mathbb{V}=\mathbb{A}^{n}$ and consider $\mathcal{P}_{d}(\mathbb{V})^{c}$ as a scheme defined over $\mathbb{Z}$. Let $T$ be the set of sequences $\left(a_{i}, b_{i}\right), 1 \leq i \leq r$, such that $0 \leq a_{i}, b_{i}<d$ and $a_{i}+b_{i} \leq d$. For any $t=\left\{\left(a_{i}, b_{i}\right)\right\} \in T$ we denote by $\nu_{t}: \oplus_{i=1}^{r} \mathcal{P}_{a_{i}}(\mathbb{V}) \otimes \mathcal{P}_{b_{i}}(\mathbb{V}) \rightarrow \mathcal{P}_{d}(\mathbb{V})$ the linear map given by

$$
\nu_{i}\left(\left\{Q_{i} \otimes R_{i}\right\}\right)=\sum_{i=1}^{r} Q_{i} R_{i} .
$$

Let $\mathbb{Z}$ be the union of images of the maps of $\nu_{t}, t \in T$.

Let $\mathbb{Y} \subset \mathcal{P}_{d}(\mathbb{V})^{c}$ be constructible subset of families $\bar{P}=\left\{P_{i}\right\}$ such that $\mathbb{Z} \cap L_{\bar{P}}=$ $\{0\}$ where $L_{\bar{P}} \subset \mathcal{P}_{d}(\mathbb{V})$ is the span of $\left(P_{i}\right)$.

So $\mathbb{Y}(k) \subset \mathcal{P}_{d}(\mathbb{V})(k)$ consists families $\bar{P}$ of polynomials of rank $>r$. We define $\mathbb{R} \subset \mathbb{Y}$ as the constructible subset of $\bar{P} \in \mathbb{Y}$ such that the morphism $\tilde{\kappa}_{\bar{P}}$ is not surjective. Our goal is to show that $\mathbb{R}=\emptyset$.

We first consider the case when $k=\overline{\mathbb{F}}_{p}$ is the algebraic closure of $\mathbb{F}_{p}$.

Claim 3.16. $\mathbb{R}\left(\overline{\mathbb{F}}_{p}\right)=\emptyset$.

Proof. Assume that $\mathbb{R}\left(\overline{\mathbb{F}}_{p}\right) \neq \emptyset$. Then there exists $\bar{P} \in \mathbb{Y}\left(\overline{\mathbb{F}}_{p}\right)$ such that the map $\tilde{\kappa}_{\bar{P}}\left(\overline{\mathbb{F}}_{p}\right)$ is not surjective. Then there exists $Q \in \mathcal{P}_{\bar{d}}\left(\overline{\mathbb{F}}_{p}\right)$ which is not in the image of $\tilde{\kappa}_{\bar{P}}\left(\overline{\mathbb{F}}_{p}\right)$. By definition there exists $l \geq 1$ such that $\bar{P} \in \mathbb{Y}\left(\overline{\mathbb{F}}_{q}\right)$ and $Q \in \mathcal{P}_{\bar{d}}\left(\mathbb{F}_{q}\right), q=p^{l}$. But as follows from Theorem 2.9 there exists $\phi \in \operatorname{Aff}_{m}\left(\mathbb{F}_{q}\right)$ such that $Q=\tilde{\kappa}_{\bar{P}}(\phi)$. But the existence of such affine map $\phi$ contradicts the assumption that $Q$ is not in the image of $\tilde{\kappa}_{\bar{P}}\left(\overline{\mathbb{F}}_{p}\right)$.

\section{Corollary 3.17.}

(1) The map $\tilde{\kappa}_{P}(k)$ is surjective for any algebraically closed field $k$ and a polynomial $P \in \mathcal{P}_{d}(\mathbb{V})$ of nc-rank $>r(m, d)$.

(2) The map $\tilde{\kappa}_{P}(k)$ is surjective for any algebraically closed field $k$ of characteristic 0 and a polynomial $P \in \mathcal{P}_{d}(\mathbb{V})$ of nc-rank $>r(m, d)$.

Proof. The part (1) follows from the completeness of the theory $A C F_{p}$ of algebraically closed fields of a fixed characteristic $p$.

To prove the part (2) one choses a non-trivial ultrafilter $\mathcal{U}$ on the set of primes and considers the $\mathcal{U}$-ultraproduct of theories $A C F_{p}$. Let $l$ be the $\mathcal{U}$-ultraproduct of fields $\overline{\mathbb{F}}_{p}$.

Let ACF be the theory of algebraically closed fields and $\alpha$ be the formula in ACF expressing the surjectivity of the map $\kappa_{P}$. As follows from Claim $3.16 \alpha$ holds for algebraic closures of fields $\mathbb{F}_{p}$. We fix now $m, d, c$ and for any $n \geq 1$ define by $\alpha_{n}$ the following formula in $A C F$. 
For any family $\bar{P}=\left(P_{i}\right), P_{i} \in k\left[x_{1} \ldots, x_{n}\right]$ such that $r_{n c}(\bar{P}) \geq r(m, d, c)$ the map $\kappa_{\bar{P}}: \operatorname{Aff}_{m}\left(\mathbb{A}^{n}\right) \rightarrow\left(\mathcal{P}_{d}\left(\mathbb{A}^{m}\right)\right)^{c}$ is surjective.

By the Theorem of Łoś applied to the formula $\alpha_{n}$ we see that the map $\kappa_{P}(l)$ is surjective for any family $\bar{P} \in \mathcal{P}_{d}(\mathbb{V}), \operatorname{dim}(\mathbb{V})=n$ of nc-rank $>r$. Since the theory $A C F_{p}$ of algebraically closed fields of characteristic 0 is complete and $n \geq 1$ is arbitrary the corollary is proved.

3.4.2. The computation of the dimensions of fibers of $\tilde{\kappa}(k)$. Let $\operatorname{Hom}_{\mathrm{af}}(\mathbb{W}, \mathbb{V})$ be the variety of affine maps from $\mathbb{W}$ to $\mathbb{V}$ and let $\mathbb{T} \subset \mathbb{Y}$ be be the subscheme of polynomials $P$ such that there exists $Q \in \mathcal{P}_{d}\left(\mathbb{A}^{m}\right)$ such that $\operatorname{dim}\left(\kappa_{P}^{-1}(Q)\right) \neq$ $\operatorname{dim}\left(\operatorname{Hom}_{\mathrm{af}}(\mathbb{W}, \mathbb{V})\right)-\operatorname{dim}\left(\mathcal{P}_{d}(\mathbb{W})\right)$.

We want to prove that $\mathbb{T}=\emptyset$. The same arguments as before show that it is sufficient to prove that

$$
\operatorname{dim}\left(\kappa_{P}^{-1}(Q)\right)=\operatorname{dim}\left(\operatorname{Hom}_{\mathrm{af}}(\mathbb{W}, \mathbb{V})\right)-\operatorname{dim}\left(\mathcal{P}_{d}(\mathbb{W})\right)
$$

for all finite fields $k=\mathbb{F}_{q}$ and $Q \in \mathcal{P}_{d}\left(\mathbb{A}^{m}\right)(k)$. Let $w:=\operatorname{dim}\left(\operatorname{Hom}_{\mathrm{af}}(\mathbb{W}, \mathbb{V})\right)-$ $\operatorname{dim}\left(\mathcal{P}_{d}(\mathbb{W})\right)$. As follows from [22] that there exists constant $A(n, d), l>0$ such that $\| \kappa_{P}^{-1}(Q)\left(\mathbb{F}_{q^{m}}\right)\left|-q^{w}\right| \leq A(n, d) q^{w-1 / 2}$ for any $q=p^{l m}$.

This implies that

$$
\operatorname{dim}\left(\kappa_{P}^{-1}(Q)\right)=\lim _{m \rightarrow \infty} \frac{\log _{q}\left(\left|\kappa_{P}^{-1}(Q)\left(k_{m}\right)\right|\right)}{\operatorname{lm}},
$$

where $k_{m}=\mathbb{F}_{q^{m l}}$ is the extension of degree $l$. Now the equality

$$
\operatorname{dim}\left(\kappa_{P}^{-1}(Q)\right)=w
$$

follows from Theorem 2.9.

Part (3) of Theorem 2.13 follows now from Theorem 23.1 in [25], and part (4) follows from the part (3).

The derivation of Theorem 2.15 from Theorem 2.11 is completely analogous.

3.5. Proof of Theorem 1.9. Proof of Theorem 1.9. Let $\mathcal{G}$ be a subfunctor of $\mathcal{F}_{d}$ such that $r(P), P \in \mathcal{G}(W)$ is not bounded above. We want to show that $\mathcal{G}(W)=\mathcal{F}_{d}(W)$ for any finite-dimensional $k$-vector space $W$.

Let $m=\operatorname{dim}(W)$ and choose a polynomial $P \in \mathcal{G}(V)$, where $V$ is a $k$-vector space $V$ such that $r_{n c}(P) \geq r(m, d)$, where $r(m, d)$ is as in the Corollary 3.17 . Then for any polynomial $Q$ on $W$ of degree $d$, there exist an affine map $\phi: W \rightarrow V$ such that $Q=\phi^{\star}(P)$. We see that $\mathcal{G}(W)=\mathcal{F}_{d}(W)$.

\section{EXTENDING WEAKLY POLYNOMIAL FUNCTIONS FROM HIGH RANK VARIETIES}

4.1. Introduction. We fix $d, a, c \geq 1$ and a field $k$ such that $|k|>a d$ and that there exists a root of unity $\beta \in k$ of order $m>a d$. A field is admissible if it satisfies these conditions. 
Definition 4.1. Let $V$ be a $k$-vector space, and let $X \subset V$. We say that a function $f: X \rightarrow k$ is weakly polynomial of degree $\leq a$ if restrictions $f_{\mid L}$ to affine subspaces $L \subset X$ are polynomials of degree $\leq a$.

Remark 4.2. If $|k|>a$ it suffices to check this on 2-dimensional subspaces (see [15], Theorem 1). Namely a function is weakly polynomial of degree $\leq a$ if the restriction $f_{\mid L}$ to 2-dimensional affine subspace $L \subset X$ is a polynomial of degree $\leq a$.

The goal of this section is to show in the case when $k$ is admissible field which is either finite or algebraically closed then any weakly polynomial function $f$ on a subvariety $X \subset V$ of a sufficiently high rank extends to a polynomial $F$ of degree $\leq a$ on $V$. The main difficulty is in the case when $a \geq d$ when an extension $F$ of $f$ is not unique.

To state our result properly we introduce the following definition:

Definition 4.3. An algebraic $k$-subvariety $\mathbb{X} \subset \mathbb{V}$ satisfies the condition $\star_{a}^{k}$ if any weakly polynomial function of degree $\leq a$ on $X$ is a restriction of a polynomial function of degree $\leq a$ on $V$.

The following example demonstrates the existence of cubic surfaces $\mathbb{X} \subset \mathbb{A}^{2}$ which do not have the property $\star_{1}^{k}$ for any field $k \neq \mathbb{F}_{2}$.

Example 4.4. Let $V=k^{2}, Q=x y(x-y)$. Then $X=X_{0} \cup X_{1} \cup X_{2}$ where $X_{0}=\{v \in V \mid x=0\}, X_{1}=\{v \in V \mid y=0\}, X_{2}=\{v \in V \mid x=y\}$. The function $f: X \rightarrow k$ such that $f(x, 0)=f(0, y)=0, f(x, x)=x$ is weakly linear but one can not extend it to a linear function on $V$.

The main result of this section is that high rank hypersurfaces over admissible fields satisfy $\star_{a}^{k}$.

Theorem 4.5. There exists an $r=r(a, d)$ such that for any admissible field $k$ which is either finite or algebraically closed, any hypersurface $\mathbb{X}$ of degree $d$ and $n c$-rank $\geq r$ in a $k$-vector space satisfies $\star_{a}^{k}$.

The result extends without difficulty to complete intersections $\mathbb{X} \subset \mathbb{V}$ of bounded degree and codimension, and high rank (see Definition 2.1).

Theorem 4.6. For any $c>0$, there exists an effective bound $r=r(a, d, c)$ such that for any admissible field $k$, which is either finite or algebraically closed, a $k$-vector space $\mathbb{V}$, any subvariety $\mathbb{X} \subset \mathbb{V}$ of codimension $c$, degree $d$ and nc-rank $\geq r$ satisfies $\star_{a}^{k}$.

Our proof of Theorem 4.6 consists of two steps.

We first construct for any $d$ hypersurfaces $\mathbb{X}_{n} \subset \mathbb{V}_{n}$ over $\mathbb{Z}$ of degree $d$ and arbitrary high rank such that for any admissible field $k$ and any $c$ the subset $\mathbb{X}_{n}(k)^{c} \subset \mathbb{V}_{n}(k)^{c}$ satisfying the conditions of Theorem 4.6. This result is purely algebraic. In the second step we show how to derive the general case of Theorem 4.6 from this special case. 
Remark 4.7. The case $a<d$ was studied in [18]. The case $a=d=2$ of was studied in [16], and a bilinear version of it was studied in [7], where it was applied as part of a quantitative proof for the inverse theorem for the $U_{4}$-norms over finite fields. We expect the results in this paper to have similar applications to a quantitative proof for the inverse theorem for the higher Gowers uniformity norms, for which at the moment only a non quantitative proof using ergodic theoretic methods exists [3, 28, 29].

4.2. Construction of an explicit collection of subvarieties. Let $\mathbb{W}:=$ $\mathbb{A}^{d}, \mathbb{V}_{n}:=\mathbb{W}^{n}$, and $P_{n}: \mathbb{V}_{n} \rightarrow \mathbb{A}$ be given by $P_{n}\left(w_{1}, \ldots, w_{n}\right)=\sum_{i=1}^{n} \mu\left(w_{i}\right)$, where $\mu: \mathbb{W} \rightarrow \mathbb{A}$ is the product $\mu\left(x^{1}, \ldots, x^{d}\right):=\prod_{j=1}^{d} x^{j}$. Let $\mathbb{X}_{n} \subset \mathbb{V}_{n}$ be the hypersurface defined by the equation $P_{n}(v)=0$.

\section{Theorem 4.8.}

(1) There exists $\epsilon>0$ such that the nc-rank $r_{n c}\left(P_{n}\right) \geq n^{\epsilon}$.

(2) For any admissible field $k$ and any $c \geq 1$ the subvariety $\left(\mathbb{X}_{n}\right)^{c} \subset \mathbb{V}^{c}$ has the property $\star_{a}$.

Remark 4.9. To simplify notations we present the proof only in the case when $c=1$. The proof in the general case is completely analogous.

\subsection{Proof of Theorem 4.8,}

4.3.1. Proof of the part (1) of Theorem 4.8. In this subsection we prove the part (1) of Theorem 4.8 .

Proof. First we note that for a non trivial character $\psi$ on $k$ we have

$$
\left|\mathbb{E}_{w \in V} \psi(\mu(w))\right|=t<1
$$

Now we observe that

$$
\left.\tilde{\mu}\left(u_{1}^{1}, \ldots, u_{d}^{1}, \ldots, u_{1}^{1}, \ldots, u_{d}^{1}\right)\right|_{\left\{u_{j}^{l}=0, l \neq j\right\}}=u_{1}^{1} u_{2}^{2} \cdots u_{d}^{d}
$$

Denote

$$
U=\left\{\left(\left(u_{1}\right)_{1}, \ldots,\left(u_{n}\right)_{1}, \ldots,\left(u_{1}\right)_{d}, \ldots,\left(u_{n}\right)_{d}\right) \in V^{d}:\left(u_{i}\right)_{j}^{l}=0, l \neq j, i \in[n]\right\}
$$

Then restricted to $U$ we have

$$
\left.\tilde{P}_{n}\left(\left(u_{1}\right)_{1}, \ldots,\left(u_{n}\right)_{1}, \ldots,\left(u_{1}\right)_{d}, \ldots,\left(u_{n}\right)_{d}\right)\right|_{U}=\sum_{i=1}^{n} \mu\left(\left(u_{i}\right)_{1}^{1},\left(u_{i}\right)_{2}^{2}, \ldots,\left(u_{i}\right)_{d}^{d}\right),
$$

so that

$$
\mathbb{E}_{u \in V^{d}} \psi\left(\tilde{P}_{n}(u)\right) \leq \mathbb{E}_{u \in U} \psi\left(\tilde{P}_{n}(u)\right)=\left|\mathbb{E}_{w \in W} \psi(\mu(w))\right|^{n}=t^{n} .
$$

It follows by 3.4 that $\tilde{P}_{n}$ is of rank $>n^{\epsilon}$ for some $\epsilon>0$.

Definition 4.10. (1) For any set $X$ we denote by $k[X]$ the space of $k$-valued functions on $X$. 
(2) For a subset $X$ of a vector space $V$, we denote by $\mathcal{P}_{a}^{w}(X) \subset k[X]$ the subspace of weakly polynomial functions of degree $\leq a$.

(3) We denote by $\mathcal{P}_{a}(X) \subset \mathcal{P}_{a}^{w}(X)$ the subspace of functions $f: X \rightarrow k$ which are restrictions of polynomial functions on $V$ of degree $\leq a$.

(4) As before we define $\mathbb{W}=\mathbb{A}^{d}, \mathbb{V}_{n}:=\mathbb{W}^{n}$ and denote by $\mu$ the product map $\mu: \mathbb{W} \rightarrow \mathbb{A}$ given by

$$
\mu\left(a^{1}, \ldots, a^{d}\right)=\prod_{s=1}^{d} a^{s} .
$$

We write elements of $V_{n}$ in the form

$$
v=\left(w_{1}, \ldots, w_{n}\right), 1 \leq i \leq n, w_{i} \in W .
$$

It is clear that Theorem 4.6 is equivalent to the following statement.

Theorem 4.11. Let $k$ be an admissible field, then $\mathcal{P}_{a}^{w}\left(X_{n}\right)=\mathcal{P}_{a}\left(X_{n}\right)$.

We fix $n$ and write $\mathbb{X}$ instead of $\mathbb{X}_{n}$, and $\mathbb{V}$ instead of $\mathbb{V}_{n}$. The proof of the part (2) of Theorem 4.8 uses the existence of a large group of symmetries of $X$, the existence of a linear subspace $L \subset V$ of large dimension and the existence of the subroup $\Delta \subset k^{\star}, \Delta \cong \mathbb{Z} / m \mathbb{Z}$ for $m>a d$.

Proof. We start the proof of Theorem 4.11 with the following result.

Claim 4.12. Let $Q$ be a polynomial of degree $\leq a d$ on $k^{N}$ such that $Q_{\mid \Delta^{N}} \equiv 0$. Then $Q=0$.

Proof. The proof is by induction on $N$. If $N=1$ then $Q=Q(x)$ is polynomial such that $Q(\delta)=0$ for $\delta \in \Delta$. Since $|\Delta|>$ ad we see that $Q=0$.

Assume that the result is known for $N^{\prime}=N-1$. Let $Q$ be a polynomial of degree $\leq a d$ on $k^{N}$ such that $Q_{\mid \Delta^{N}} \equiv 0$. By induction we see that $Q\left(\delta, x_{2}, \ldots, x_{s}\right) \equiv 0$ for all $\delta \in \Delta$. Then for any $x_{2}, \ldots, x_{s}$ the polynomial $x \rightarrow Q\left(x, x_{2}, \ldots, x_{s}\right)$ vanishes for all $\delta \in \Delta$. Therefore $Q\left(x, x_{2}, \ldots, x_{s}\right)=0$ for all $x \in k$.

\section{Definition 4.13.}

(1) $\Gamma:=\left(S_{d}\right)^{n}$. The group $\Gamma$ acts naturally on $X$.

(2) $L:=\left\{\left(c_{1}, \ldots, c_{n}\right) \in k^{n} \mid \sum_{i=1}^{n} c_{i}=0\right\}$.

(3) $L_{\Delta}=(\Delta)^{n} \cap L \subset k^{n}$.

(4) For $c \in k$ we write $w(c):=(c, 1, \ldots, 1) \in W$.

(5) $\kappa: L \hookrightarrow X \subset V$ is the linear map given by

$$
\kappa\left(c_{1}, \ldots, c_{n}\right):=\left(w\left(c_{1}\right), \ldots, w\left(c_{n}\right)\right)
$$

and write $\kappa_{\gamma}:=\gamma \circ \kappa, \gamma \in \Gamma$.

(6) For any function $f: X \rightarrow k, \gamma \in \Gamma$ define a function $h_{\gamma, f}: L \rightarrow k$ by $h_{\gamma, f}:=f \circ \kappa_{\gamma}$.

(7) $T_{1}:=\left\{\left(u_{1}, \ldots, u_{d}\right) \in(\Delta)^{d} \mid \prod_{j=1}^{d} u_{j}=1\right\}$ and $T:=T_{1}^{n}$. 
(8) We denote by $\zeta_{i}: T_{1} \hookrightarrow T, 1 \leq i \leq n$ the imbedding onto the i-th component.

(9) For any $j, j^{\prime}, 1 \leq j \neq j^{\prime} \leq d$ we denote by $\phi_{j, j^{\prime}}: \Delta \rightarrow T_{1}$ the morphism such that $\phi_{j, j^{\prime}}(u)=\left(x_{l}(u), 1 \leq l \leq d\right)$ where $x_{j}(u)=u, x_{j^{\prime}}(u)=u^{-1}$ and $x_{l}(u)=1$ for $l \neq j, j^{\prime}$.

(10) We denote by $\Theta_{1}$ the group of homomorphisms $\chi: T_{1} \rightarrow k^{\star}$.

(11) $\Theta=\left(\Theta_{1}\right)^{n}$.

(12) For $\chi \in \Theta_{1}, j, j^{\prime}, 1 \leq j \neq j^{\prime} \leq d$ we define a homomorphism $\chi_{j, j^{\prime}}: \Delta \rightarrow k^{\star}$ by $\chi_{j, j^{\prime}}:=\chi \circ \phi_{j, j^{\prime}}$. Since $\Delta \cong \mathbb{Z} / m \mathbb{Z}$ there exists unique $\alpha_{j, j^{\prime}}(\chi) \in$ $(-m / 2, m / 2]$ such that $\chi_{j, j^{\prime}}(u)=u^{\alpha_{j, j^{\prime}}(\chi)}$ for any $u \in \Delta$.

(13) $\Theta_{1}^{a d m}:=\left\{\chi \in \Theta_{1}:\left|\alpha_{j, j^{\prime}}(\chi)\right| \leq a\right\}$.

(14) $\Theta_{1}^{a d m,+}:=\left\{\chi \in \Theta_{1}^{a d m}: \alpha_{j, j^{\prime}}(\chi) \geq 0, j<j^{\prime}\right\}$.

(15) Let $\Theta^{a d m,+}:=\left(\Theta_{1}^{a d m,+}\right)^{n}$ and $\Theta^{a d m}:=\left(\Theta_{1}^{a d m}\right)^{n}$.

(16) For any $k$-vector space $R$, a representation $\pi: T \rightarrow \operatorname{Aut}(R)$ and $\theta \in \Theta$ we define

$$
R^{\theta}=\{r \in R \mid \pi(t) r=\theta(t) r, t \in T\} .
$$

Remark 4.14. Since $|T|$ is prime to $q:=\operatorname{char}(k)$ the Maschke's theorem implies the direct sum decomposition $R=\oplus_{\theta \in \Theta} R^{\theta}$.

Claim 4.15. For any $f \in \mathcal{P}_{a}^{w}(X), \gamma \in \Gamma$ the function $h_{\gamma, f}$ is a polynomial of degree $\leq a$.

Proof. Since $f \in \mathcal{P}_{a}^{w}(X)$ we have $h_{\gamma, f} \in \mathcal{P}_{a}^{w}(L)$. Since $L$ is linear space we see that $h_{\gamma, f}$ is a polynomial of degree $\leq a$.

\section{Claim 4.16.}

(1) The subset $\Theta^{a d m}$ of $\Theta$ is $\Gamma$-invariant.

(2) For any $\theta \in \Theta^{a d m}$ there exists $\gamma \in \Gamma$ such that $\theta \circ \gamma \in \Theta^{a d m,+}$.

Proof. Clear.

Definition 4.17. We denote by $\mathcal{P}_{a}^{\bar{w}}(X)$ the space of functions $f$ such that $h_{\gamma, f}$ is a polynomial of degree $\leq a$ on $L$ for all $\gamma \in \Gamma$.

The group $T$ acts naturally on $X$, and on the spaces $\mathcal{P}_{a}^{\bar{w}}(X)$ and $\mathcal{P}_{a}(X)$, and we have direct sum decompositions

$$
\mathcal{P}_{a}^{w}(X)=\oplus_{\theta \in \Theta} \mathcal{P}_{a}^{w}(X)^{\theta}
$$

and

$$
\mathcal{P}_{a}(X)=\oplus_{\theta \in \Theta} \mathcal{P}_{a}(X)^{\theta} .
$$

Lemma 4.18. Let $\mathbb{Z} \subset \mathbb{V}$ be a homogeneous $k$-subvariety of degree $d$ and let $f: Z \rightarrow k$ a polynomial function of degree ad which is a weakly polynomial function on $Z$ of degree $\leq a$. Then it is a restriction of polynomial function on $V(k)$ of degree $\leq a$. 
Proof. Lemma 4.18 follows inductively from the following claim.

Claim 4.19. Let $f: Z \rightarrow k$ be a polynomial function of degree $\leq a$ which is a weakly polynomial of degree $<a$. Then $f$ is a polynomial of degree $<a$.

Proof. We can write $f$ as a sum $f=Q+f^{\prime}$ where f' $<a$ and $Q$ is homogeneous of degree $a$. Since $f$ is weakly polynomial of degree $<a$ the function $Q$ is also weakly polynomial of degree $<a$. It is sufficient to show that $Q \equiv 0$.

Choose $z \in Z$. To show that $Q(z)=0$ consider the function $g$ on $k, g(t)=$ $Q(t z)$. Since $Z$ is homogeneous $t z \in Z$. Since $Q$ is homogeneous of degree $a$ we have $g(t)=c t^{a}$. On the other hand, since $Q$ is weakly polynomial of degree $<a$ we see that $g(t)$ is a polynomial of degree $<a$. Since $a<q$ we see that $g \equiv 0$. So $Q(z)=g(1)=0$.

Corollary 4.20. Let $f: X \rightarrow k$ be a weakly polynomial of degree $<a$ on $X$ which is a restriction of polynomial function of degree $\leq$ ad on $V$. Then $f$ is a restriction of polynomial function of degree $\leq a$ on $V$.

It is clear that for a proof Theorem 4.11 it suffices to show that $\mathcal{P}_{a}^{w}(X)^{\theta}=$ $\mathcal{P}_{a}(X)^{\theta}$ for any $\theta \in \Theta$. This equality follows now immediately from the following statement. Fix $\theta \in \Theta$.

Proposition 4.21. For any weakly polynomial function $f: X \rightarrow k$ of degree $\leq a$ satisfying the equation $f(t x)=\theta(t) f(x), t \in T, x \in X$ there exists a polynomial $P$ on $V$ of degree $\leq$ ad such that $f=P_{\mid X}$.

We start the proof of Proposition 4.21 with a set of definitions. Let $f: X \rightarrow k$ be a function such that $f(t x)=\theta(t) f(x), t \in T, x \in X$, and such that $h_{\gamma, f}$ are polynomial functions on $L$ of degree $\leq a$ for all $\gamma \in \Gamma$.

\section{Definition 4.22.}

(1) We write $h, h_{\gamma}: L \rightarrow k$ instead of $h_{\mathrm{Id}, f}$ and $h_{\gamma, f}$.

(2) $\nu: V \rightarrow L$ is the map given by $\nu\left(w_{1}, \ldots, w_{n}\right):=\left(\mu\left(w_{1}\right), \ldots, \mu\left(w_{n}\right)\right)$.

(3) $W^{0}:=\left\{w=\left(a^{1}, \ldots, a^{d}\right) \in W \mid a^{i} \in \Delta\right.$ for $\left.i \geq 2\right\} \subset W$.

(4) $X^{0}:=\left(W^{0}\right)^{n} \cap X$.

(5) For $w=\left(a^{1}, \ldots, a^{d}\right) \in W$ we define $I(w):=\left\{i, 1 \leq i \leq d \mid a^{i} \notin \Delta\right\}$ and write $z(w):=\max (|I(w)|-1,0)$.

(6) For $x=\left(w_{1}, \ldots, w_{n}\right)$ we write $z(x)=\sum_{j} z\left(w_{j}\right)$.

(7) $Y_{s}:=\{x \in X \mid z(x) \leq s\}, s \geq 0$.

\section{Claim 4.23.}

(1) $Y_{0}=\left\{\gamma\left(W^{0}\right), \gamma \in \Gamma\right\}$.

(2) For any $x \in X^{0}$ there exist unique $t(x) \in T$ such that $x=t(x) \kappa(\nu(x))$.

(3) $f(x)=\theta(t(x)) f(\kappa(\nu(x)))$ for any $x \in X^{0}$. 
(4) For any $\gamma \in \Gamma, l \in L$, we have $\nu(\gamma(l))=l$ where $\gamma: X \rightarrow X$ is as in Definition 4.13 ,

Proof. Clear.

Lemma 4.24. Let $f$ be a function on $X$ satisfying the conditions of Proposition 4.21 and such that $f_{\mid Y_{0}} \equiv 0$. Then $f \equiv 0$.

Proof. It is clear that it is sufficient to prove the following statement

Claim 4.25. Let $f$ be a function on $X$ satisfying the conditions of Proposition 4.21 and such that $f_{\mid Y_{s}} \equiv 0, s \geq 0$. Then $f_{\mid Y_{s+1}} \equiv 0$.

Proof. We want to show that $f(x)=0$ for all $x=\left(w_{j}\right) \in Y_{s+1}$. Since the restriction of $f$ on any line is a polynomial of degree $\leq a d$ it is clear that for a proof of the equality $f(x)=0$ it is sufficient to prove the following geometric statement.

Claim 4.26. There exists a line $N \subset X$ containing $x$ and such that $\left|N \cap Y_{s}\right|>a d$.

Proof. Let $x=\left(w_{j}\right), w_{j}=\left(x_{j}^{1}, \ldots, x_{j}^{d}\right), 1 \leq j \leq n$. We start with the following observation.

Claim 4.27. For any $x \in X \backslash Y_{0}, x=\left\{x_{j}^{i}\right\}$ there exists $j_{0}$ such that either there exist $\left(i_{0}, i_{1}\right), 1 \leq i_{0} \neq i_{1} \leq d$ such that $x_{j_{0}}^{i_{0}}=0, x_{j_{0}}^{i_{1}} \notin \Delta$ or $\prod_{i} x_{j_{0}}^{i} \neq 0$ and $x_{j_{0}}^{i_{0}} \notin \Delta$ for some $i_{0}, 1 \leq i_{0} \leq d$.

Proof. Clear.

Consider first the case when $x_{j_{0}}^{i_{0}}=0$ for some pair $\left(i_{0}, j_{0}\right), 1 \leq i_{0} \leq d, 1 \leq j_{0} \leq n$ and $x_{j_{0}}^{i_{1}} \notin \Delta$ for some $i_{1} \neq i_{0}, 1 \leq i_{0} \leq d$. Let $\alpha: k \rightarrow X$ be the map given by $\alpha(c)=x_{j}^{i}(c)$ where $x_{j}^{i}(c)=x_{j}^{i}$ if $(i, j) \neq\left(i_{1}, j_{0}\right)$ and $x_{j_{0}}^{i_{1}}(c)=c$. By construction $x \in N:=\operatorname{Im}(\alpha)$ and $x_{j_{0}}^{i_{1}}(\Delta) \subset Y_{s}$ for $c \in \Delta$. Since $|\Delta|=q>a d$ we see that the line $N$ satisfies the conditions of Claim 4.26,

So we may assume the existence of $j_{0}$ such $\prod_{i} x_{j_{0}}^{i} \neq 0$ and $x_{j_{0}}^{i_{0}} \notin \Delta$ for some $i_{0}, 1 \leq i_{0} \leq d$. To simplify notations we may and will assume that $j_{0}=i_{0}=1$.

Since $x \in X$ there exists $j_{1}, 2 \leq j \leq n$ such that $\prod_{i} x_{j_{1}}^{i} \neq 0$. We may assume that $j_{1}=2$. It is clear that either $x_{2}^{i} \in \Delta$ for all $i, 1 \leq i \leq d$ or there exists $i_{1}, 1 \leq i_{1} \leq d$ such that $x_{2}^{i_{1}} \notin \Delta$ in which case we may and will assume that $i_{1}=1$.

Let $a:=\prod_{i=2}^{d} x_{1}^{i}, b:=\prod_{i=2}^{d} x_{2}^{i}$ and $\beta: k \rightarrow X$ be the map given by $\beta(c):=$ $x_{j}^{i}(c)$ where $x_{1}^{1}(c)=-b c, x_{1}^{2}(c)=a c-\sum_{j=3}^{n} \prod_{i=1}^{d} x_{j}^{i}$ and $x_{j}^{i}(c)=x_{j}^{i}$ otherwise. Let $a:=\prod_{i=2}^{d} x_{1}^{i}, b:=\prod_{i=2}^{d} x_{2}^{i}$ and $\beta: k \rightarrow X$ be the map given by $\beta(c):=x_{j}^{i}(c)$ where $x_{1}^{1}(c)=-b c, x_{2}^{1}(c)=a c-b^{-1} \sum_{j=3}^{n} \prod_{i=1}^{d} x_{j}^{i}$ and $x_{j}^{i}(c)=x_{j}^{i}$ otherwise. Let $N=\operatorname{Im}(\beta)$. Then $x \in N$ and $\left|N \cap Y_{s}\right|=q>a d$. 
Lemma 4.28. $\mathcal{P}_{a}^{\bar{w}}(X)^{\theta}=\{0\}$ for any $\theta \notin \Theta^{a d m}$.

Proof. As follows from Lemma 4.24 it is sufficient to show that $h_{\gamma}(f) \equiv 0$ for any $\theta \notin \Theta^{a d m}, \gamma \in \Gamma$ and $f \in \mathcal{P}_{a}^{\bar{w}}(X)^{\theta}$. Since $\theta \notin \Theta^{a d m}$ there exist $i, j, j^{\prime}, 1 \leq i \leq$ $n, 1 \leq j, j^{\prime} \leq d$, such that $\left|\alpha_{j, j^{\prime}}\left(\chi_{i}\right)\right| \geq a$. Choose $s \in S_{d}$ such $s(j)=1, s\left(j^{\prime}\right)=2$, and denote by $\tilde{s} \in \Gamma$ the image of $s$ under the imbedding $S_{d} \hookrightarrow \Gamma$ as the $i$-factor. After the replacing $f \rightarrow f \circ \tilde{s}, \theta \rightarrow \theta \circ s$ we may assume that $\left|\alpha_{1,2}\left(\chi_{i}\right)\right|>a$.

The functions $h_{\gamma}$ and $h_{\gamma \circ s}$ are weakly polynomial functions of degrees $\leq a$ on the linear space $L$. Therefore $h_{\gamma}$ and $h_{\gamma \circ s}$ are polynomial functions of degrees $\leq a$.

For any $l \in L$ such that $l_{i} \in \Delta$ we have $h_{\gamma \circ s}(l)=l_{i}^{\alpha_{1,2}\left(\chi_{i}\right)} h_{\gamma}(l)$. Since $\alpha_{1,2}\left(\chi_{i}\right)>$ $a$ and $\alpha_{1,2}\left(\chi_{i}\right) \leq m / 2$, this is only possible if $h_{\gamma}=0$.

Corollary 4.29. As follows from Claim 4.16 it is sufficient to prove Proposition 4.21 for $\theta \in \Theta^{\text {adm, }+}$.

Definition 4.30. Let $P: V \rightarrow k$ be the polynomial given by

$$
P(v)=\prod_{i=1}^{n} \prod_{j=1}^{d}\left(x_{i}^{j}\right)^{\alpha^{1, j}\left(\chi_{i}\right)} h(\nu(v)), \quad v=\left(x_{i}^{j}\right) .
$$

Lemma 4.31. $\operatorname{deg}(P) \leq a d$.

Proof. Let $b=\operatorname{deg}(h)$. It is sufficient to show that for any sequence $\bar{e}=$ $(e(i))_{1 \leq i \leq n}, e(i) \in[1, d]$, we have $\sum_{i=1}^{n} \alpha^{1, e(i)}\left(\chi_{i}\right)+b \leq a$.

Suppose there exists $\bar{e}$ such that $\sum_{i=1}^{n} \alpha^{1, e(i)}\left(\chi_{i}\right)+b>a$. Since $\theta \in \Theta_{n}^{a d m}$, there exists a subset $I$ of $[1, n]$ such that $a<\sum_{i \in I} \alpha^{1, e(i)}\left(\chi_{i}\right)+b \leq 2 a$.

Let $\gamma=\left(\sigma_{i}\right)_{1 \leq i \leq n}, \sigma_{i} \in S_{d}$, be such that $e(i)=\sigma_{i}(1)$ for $i \in I$ and $\sigma_{i}=I d$ if $i \notin I$. Consider $h_{\gamma}:=\kappa_{\gamma}^{\star}(f)$. On one hand it is a polynomial of degree $\leq a$ on $L$.

On the other $h_{\gamma}(l)=h(l) \prod_{i \in I} l_{i}^{\alpha^{1, e(i)}}\left(\chi_{i}\right)$. The inequalities $a<\sum_{i \in I} \alpha^{1, e(i)}\left(\chi_{i}\right)+$ $b \leq 2 a$ imply that $h \equiv 0$.

By construction $P_{\mid L} \equiv f_{\mid L}$. Let $\bar{f}:=f-P$. Then $\bar{f}$ is weakly polynomial function of degree $\leq a d$ on $X$ vanishing on $L$ such that $\bar{f}(t x)=\theta(t) f(x)$ for $t \in T, x \in X$. As follows from Claim 4.23 we have $\bar{f}_{\mid X^{0}} \equiv 0$. It is clear that for a proof the part (1) of Proposition 4.21 it is sufficient to show that $\bar{f}(x)=0$ for all $x \in X$. By Lemma 4.24 it suffices to prove the following lemma:

Lemma 4.32. $\bar{f}_{\mid Y_{0}} \equiv 0$.

Proof. Since $Y_{0}=\Gamma X^{0}=T L_{\gamma}$, it suffices to show that $f_{\mid L_{\gamma}} \equiv 0$ for all $\gamma \in \Gamma$. Let $h_{\gamma}: L \rightarrow k$ be given by $h_{\gamma}(l)=\bar{f}(\gamma(l))$. We have to show that $h_{\gamma} \equiv 0$. Since $h_{\gamma}$ is a polynomial of degree $\leq a d$ it follows from Claim 4.12 that it is sufficient to show that the restriction of $h_{\gamma}$ on $L_{\Delta}$ vanishes. But for any $l \in L_{\Delta}$ we have $\gamma(l)=t l^{\prime}, t \in T, l^{\prime} \in L$. Since $\bar{f}(t x)=\theta(t) f(x)$ we see that $h_{\gamma}(l)=0$. 
This completes the proof of Theorem 4.11.

\subsection{Proof of Theorem 4.6.}

Proposition 4.33. There exists an effective bound $r=r(\bar{d}, a)$ such that if $k$ is an admissible field which is either finite or algebraically closed, $W \subset V$ is a hyperplane then any weakly polynomial function on $X_{\bar{P}}, r_{n c}(\bar{P}) \geq r$ of degree $\leq a$ vanishing on $X_{\bar{P}} \cap W$ is a restriction of a polynomial on $V$ of degree $\leq a$.

As an immediate corollary we obtain:

Corollary 4.34. There exists an effective bound $r=r(\bar{d}, a)$ such that the following holds for all admissible fields $k$ which are either finite or algebraically closed.

Let $\mathbb{X}=\left\{v \in \mathbb{V} \mid P_{i}(v)=0\right\} \subset \mathbb{V}$ be a subvariety of degree $\leq d$ and $\mathbb{W} \subset \mathbb{V}$ an affine subspace such that nc-rank of $\bar{P}_{\mid \mathbb{W}} \geq r$. Let $f$ be a weakly polynomial function on $X$ of degree $\leq$ a such that $f_{\mid X \cap W}$ extends to a polynomial on $W$ of degree $\leq a$. Then there exists an extension $F$ of $f$ to a polynomial on $V$ of degree $\leq a$.

Proof. Consider first the case when $W \subset V$ is a hyperplane. By the assumption there exists an extension $R$ of the restriction $\left.f\right|_{W}$. Choose a linear projection $s: V \rightarrow W$ and $f^{\prime}: X \rightarrow k$ by $f^{\prime}(x)=f(x)-R(s(x))$. Then $f^{\prime}$ is weakly polynomial function on $X$ of degree $\leq a$ such that $f_{\mid X \cap W}^{\prime} \equiv 0$. As follows from Proposition 4.33 there exists an extension of $f^{\prime}$ to a polynomial $F^{\prime}$ on $V$ of degree $\leq a$. But then $F:=F^{\prime}+R \circ s$ is an extension $F$ of $f$ to a polynomial on $V$ of degree $\leq a$.

In the case when the codimension of $W$ is $>1$ we choose a flag

$$
\mathcal{F}=\left\{W_{0}=W \subset W_{1} \cdots \subset W_{\operatorname{dim}(V)-\operatorname{dim}(W)}=V\right\}, \operatorname{dim}\left(W_{i}\right)=\operatorname{dim}(W)+i,
$$

and extend $f$ by induction in $i, 1 \leq i \leq \operatorname{dim}(V)-\operatorname{dim}(W)$ to a polynomial $F$ on $V$.

Remark 4.35. The choice of $F$ depends on a choice of flag $\mathcal{F}$ and on choices of projections used in the inductive arguments.

4.5. Proof of Proposition 4.33. The key tool in our proof of this proposition is a testing result from [18] which roughly says that any weakly polynomial function of degree $a$ on $X$ that is "almost" weakly polynomial of degree $<a$, namely it is a polynomial of degree $<a$ on almost all affine subspaces, is weakly polynomial of degree $<a$. This part does not require $X$ to be of high rank. We use the assumption that $X$ is of high rank to show (see Theorem 2.11) that almost any isotropic affine plane is contained in an isotropic three affine dimensional subspace that is not contained in $l^{-1}\{0\}$.

We start by stating the testing result from [18]. In [15] (Theorem 1) the following description of degree $\leq$ polynomials is given: 
Proposition 4.36. Let $P: V \rightarrow k$. Then $P$ is a polynomial of degree $\leq a$ if and only if the restriction of $P$ to any affine subspace of dimension $l=\left\lceil\frac{a+1}{q-q / p}\right\rceil$ is a polynomial of degree $\leq a$.

Note that when $a<q$ then $l \leq 2$.

In [15] the above criterion is used for polynomial testing over general finite fields. In [18] (Corollary 1.13) it is shown how the arguments in [15] can be adapted to polynomial testing within a subvariety variety $X \subset V$ (high rank is not required).

Proposition 4.37 (Subspace splining on $X$ ). For any $a, d, c>0$ there exists an $A=A(d, c, a)>0$, depending polynomially on $c, d$ and exponentially on $a$, such that the following holds. Let $X \subset V(k)$ be a complete intersection of degree $d$, codimension $c$. Then any weakly polynomial function $f$ of degree a such that the restriction of $f$ to $q^{-A}$-a.e l-dimensional affine subspace, $l=\left\lceil\frac{a}{q-q / p}\right\rceil$, is a polynomial of degree $<a$, is weakly polynomial of degree $<a$.

Proof of Proposition 4.33. Let $V$ be a vector space and $l: V \rightarrow k$ be a nonconstant affine function. For any subset $I$ of $k$ we denote $\mathbb{W}_{I}=\{v \in \mathbb{V} \mid l(v) \in I\}$, so that $\mathbb{W}_{b}=\mathbb{W}_{\{b\}}$, for $b \in k$. For a hypersurface $\mathbb{X} \in \mathbb{V}$ we write $\mathbb{X}_{I}=\mathbb{X} \cap \mathbb{W}_{I}$.

Lemma 4.38. For any finite subset $S \subset k$, any weakly polynomial function $f$ of degree $a$ on $X$ such that $f_{\mid X_{S}} \equiv 0$, and any $b \in k \backslash S$, there exists a polynomial $Q$ of degree $\leq a$ on $V$ such that $Q_{\mid X_{S}} \equiv 0$ and $(Q-f)_{\mid X_{b}} \equiv 0$.

Proof. We start with the following result.

Claim 4.39. Under the assumptions of Lemma 4.38, the restriction $f_{\mid X_{b}}$ is a weakly polynomial function of degree $\leq a-|S|$.

Proof. Since $|k|>a$ it suffices to show that for any plane $L \subset X_{b}$ the restriction $f_{\mid L}$ is a polynomial of degree $\leq a-|S|$.

We first do the case when the field $k$ is finite. Since by the part (4) of Theorem 2.13 the variety $\mathbb{X}$ is a complete intersection it follows from Proposition 4.37, there is a constant $A=A(d, a)$ such that it suffices to check the restriction $f_{L}$ on $q^{-A}$-almost any affine plane $L \subset X_{b}$ is a polynomial of degree $\leq a-|S|$.

As follows from Proposition 3.12, for any $s>0$ there is an $r=r(d, s)$ such that if $X$ is of nc-rank $>r$ then for $q^{-s}$-almost any affine plane $L \subset X_{b}$ there exists an affine 3 -dim subspace $M \subset X$ containing $L$ and such that $M \cap X_{0} \neq \emptyset$. Then $M \cap X_{t} \neq \emptyset$ for any $t \in k$. Since $f$ is a weakly polynomial function of degree $a$, its restriction to $M$ is a polynomial $R$ of degree $\leq a$. Since the restriction of $R$ to $l^{-1}(S) \cap M$ is identically zero, we see that $R=R^{\prime} \prod_{s \in S}(l-s)$. Since $\left.l\right|_{L} \equiv b$, we see that the restriction $\left.f\right|_{L}$ is equal to $R^{\prime}$, which is a is a polynomial of degree $\leq a-|S|$. 
Now we show that this claim implies Lemma 4.38. Indeed, assume that $f_{\mid X_{b}}$ is a weakly polynomial function of degree $\leq a-|S|$. It follows from the inductive assumption on $a$, that there exists a polynomial $Q^{\prime}$ of degree $\leq a-|S|$ on $V$, such that $f_{\mid X_{b}}=Q_{\mid X_{b}}^{\prime}$. Let $Q:=\frac{Q^{\prime} \prod_{s \in S}(l-s)}{\prod_{s \in S}(b-s)}$. Then $(f-Q)_{X_{S \cup\{b\}}} \equiv 0$.

For algebraically closed fields we follow the same argument replacing Proposition 4.37 with Proposition A.6, and Proposition 3.12, with Theorem 2.15.

Proposition 4.33 follows from Lemma 4.38 by induction.

Now we can prove Theorem 4.5.

Proof of Theorem 4.5 assuming Theorems 4.8 and 1.9, and Corollary 4.34. Let $\tilde{r}$ be from Corollary 4.34 and $r=r(a, \bar{d}):=\rho(\operatorname{dim}(W), d)$ from Theorem 1.9, As follows from Theorem 4.8 the subvarieties $\mathbb{X}_{n}$ are of nc-rank $\geq \tilde{r}$ for $n \geq d \tilde{r}$.

Let $\mathbb{X} \subset \mathbb{V}$ be a subvariety of nc-rank $\geq r$. By Theorem 1.9 there exists a linear map $\phi: \mathbb{W} \rightarrow \mathbb{V}$ such that $\mathbb{X}_{n}=\{w \in \mathbb{W} \mid \phi(w) \in \mathbb{X}\}$. Since $\mathbb{X}_{n}$ satisfies $\star_{a}^{k}$, Corollary 4.34 implies that $\mathbb{X}$ satisfies $\star_{a}^{k}$.

\section{Nullstellensatz}

Let $k$ be a field and $V$ be a finite dimensional $k$-vector space. We denote by $\mathbb{V}$ the corresponding $k$-scheme, and by $\mathcal{P}(V)$ the algebra of polynomial functions on $\mathbb{V}$ defined over $k$.

For a finite collection $\bar{P}=\left(P_{1}, \ldots, P_{c}\right)$ of polynomials on $\mathbb{V}$ we denote by $J(\bar{P})$ the ideal in $\mathcal{P}(V)$ generated by these polynomials, and by $\mathbb{X}_{\bar{P}}$ the subscheme of $\mathbb{V}$ defined by this ideal.

Given a polynomial $R \in \mathcal{P}(\mathbb{V})$, we would like to find out whether it belongs to the ideal $J(\bar{P})$. It is clear that the following condition is necessary for the inclusion $R \in J(\bar{P})$.

(N) $R(x)=0$ for all $k$-points $x \in X_{\bar{P}}(k)$.

Proposition 5.1 (Nullstellensatz). Suppose that the field $k$ is algebraically closed and the scheme $\mathbb{X}_{\bar{P}}$ is reduced. Then any polynomial $R$ satisfying the condition $(N)$ lies in $J(\bar{P})$.

We will show that the analogous result hold for $k=\mathbb{F}_{q}$ if $\mathbb{X}_{\bar{P}}$ is of high $n c$-rank.

From now on we fix a degree vector $\bar{d}=\left(d_{1}, \ldots, d_{c}\right)$ and write $D:=\prod_{i=1}^{c} d_{i}$. We denote by $\mathcal{P}_{\bar{d}}(\mathbb{V})$ the space of $\bar{d}$-families of polynomials $\bar{P}=\left(P_{i}\right)_{i=1}^{c}$ on $V$ such that $\operatorname{deg}\left(P_{i}\right) \leq d_{i}$.

Theorem 5.2. There exists and an effective bound $r(\bar{d})>0$ such that for any finite field $k=\mathbb{F}_{q}$ with $q>a D$, any family $\bar{P}$ of degrees $\bar{d}$ and nc-rank $\geq r(\bar{d})$ the following holds. Any polynomial $Q$ on $V$ of degree a vanishing on $X_{\bar{P}}$ belongs to the ideal $J(\bar{P})$. 
Proof. Our proof is based on the following rough bound (see [11]).

Lemma 5.3. Let $\bar{P}=\left\{P_{i}\right\}_{i=1}^{c} \subset \mathbb{F}_{q}\left[x_{1}, \ldots, x_{n}\right]$ be a family of polynomials of degrees $d_{i}, 1 \leq i \leq c$ such that the variety $\mathbb{Y}:=\mathbb{X}_{\bar{P}} \subset \mathbb{A}^{n}$ is of dimension $n-c$. Then $\left|\mathbb{Y}\left(\mathbb{F}_{q}\right)\right| \leq q^{n-c} D$ where $D:=\prod_{i=1}^{c} d_{i}$.

For the convenience we reproduce the proof of this result.

Proof. Let $F$ be the algebraic closure of $\mathbb{F}_{q}$. Then $\mathbb{Y}\left(\mathbb{F}_{q}\right)$ is the intersection of $\mathbb{Y}$ with hypersurfaces $Y_{j}, 1 \leq j \leq n$ defined by the equations $h_{j}\left(x_{1}, \ldots, x_{n}\right)=0$ where $h_{j}\left(x_{1}, \ldots, x_{n}\right)=x_{j}^{q}-x_{j}$.

Let $H_{1}, \ldots, H_{n-c}$ be generic linear combinations of the $h_{j}$ with algebraically independent coefficients from an transcendental extension $F^{\prime}$ of $F$ and $\mathbb{Z}_{1}, \ldots, \mathbb{Z}_{n-c} \subset$ $\mathbb{A}^{n}$ be the corresponding hypersurfaces.

Intersect successively $\mathbb{Y}$ with $\mathbb{Z}_{1}, \mathbb{Z}_{2}, \ldots, \mathbb{Z}_{n-c}$. Inductively we see that for each $j \leq n-c$, each component $C$ of the intersection $\mathbb{Y} \cap \mathbb{Z}_{1} \cap \cdots \cap \mathbb{Z}_{j}$ has dimension $n-c-j$. Really passing from $j$ to $j+1$ for $j<n-c$ we have $\operatorname{dim}(C)=n-c-i>0$. So not all the functions $h_{j}$ vanish on $C$. Hence by the genericity of the choice of linear combinations $\left\{H_{j}\right\}$ we see that $H_{j+1}$ does not vanish on $C$ and therefore $\mathbb{Z}_{j+1} \cap C$ is of pure dimension $n-c-j-1$. Thus the intersection $\mathbb{Y} \cap \mathbb{Z}_{1} \cap \cdots \cap \mathbb{Z}_{n-c}$ has dimension 0. By Bezout's theorem we see that $\left|\mathbb{Y} \cap \mathbb{Z}_{1} \cap \ldots \mathbb{Z}_{n-c}\right| \leq q^{n-c} D$. Since $\mathbb{Y}\left(\mathbb{F}_{q}\right)=\mathbb{Y} \cap \mathbb{Z}_{1} \cap \cdots \cap \mathbb{Z}_{n} \subset \mathbb{X} \cap \mathbb{Y}_{1} \cap \cdots \cap \mathbb{Y}_{n-c}$ we see that $\left|\mathbb{Y}\left(\mathbb{F}_{q}\right)\right| \leq$ $q^{n-c} D$.

Now we can finish the proof of Theorem 5.2. Let $R \in \mathbb{F}_{q}\left[x_{1}, \ldots, x_{n}\right]$ be a polynomial of degree $a$ vanishing on the set $\mathbb{X}_{\bar{P}}\left(\mathbb{F}_{q}\right)$. Suppose that $R$ does not lie in the ideal generated by the $P_{i}, 1 \leq i \leq c$. Let $\mathbb{Y}:=\mathbb{X}_{\bar{P}}$. Since $R$ vanishes on $\mathbb{X}_{\bar{P}}\left(\mathbb{F}_{q}\right)$ we have $\mathbb{Y}\left(\mathbb{F}_{q}\right)=\mathbb{X}_{\bar{P}}\left(\mathbb{F}_{q}\right)$.

Since $\mathbb{X}_{\bar{P}}$ is irreducible we have $\operatorname{dim}(\mathbb{Y})=n-c-1$. As follows from 5.3 we have the upper bound $\left|\mathbb{Y}\left(\mathbb{F}_{q}\right)\right| \leq a D q^{n-c-1}$. On the other hand as follows from Theorem 3.2 there exists an effective bound $r(\bar{d})>0$ such that the condition $r_{n c}(\bar{P}) \geq r(\bar{d})$ implies the inequality $\left|\mathbb{X}_{\bar{P}}\left(\mathbb{F}_{q}\right)\right|>q^{n-c \frac{q-1}{q}}$, which is a contradiction to $q>a D$.

\section{Appendix A. An Algebro-Geometric analogue of Proposition 18}

Proposition 4.37 was proved in [18. In this Appendix we present another approach leading to a proof of Proposition 4.37 which (after some adjustments) provides a proof of an Algebro-Geometric analogue of Proposition 4.37.

We start with two auxiliary results.

Definition A.1. Let $A$ be a finite set, $B \subset A \times A, Z \subset A$, and let $p_{1}, p_{2}: B \rightarrow A$ be the natural projections. For $x \in A$ we define $B_{x}:=p_{2}\left(p^{-1}\right)(x) \subset A$. Let $\beta(x):=\left|B_{x}\right| /|A|$.

(1) $B$ is $\delta$-dense if $\beta(x) \geq \delta, x \in A$. 
(2) A subset $C \subset A$ is $\epsilon$-invariant if $\left|B_{z} \cap C\right| /\left|B_{z}\right| \geq 1-\epsilon, z \in C$.

Claim A.2. If $B$ is $\delta$-dense, $C \subset A$ is $\epsilon$-invariant, and $|C| /|A|<\delta(1-\epsilon)$, then $C=\emptyset$.

Proof. Let $\alpha=|A|, \beta=|B|, \gamma=|C|$. Since

$$
\bigcup_{z \in C} z \times B_{z} \cap C \subset C \times C
$$

we have $\gamma^{2} \geq \alpha \gamma \delta(1-\epsilon)$. Since $\gamma / \alpha<\delta(1-\epsilon)$, we see that $\gamma=0$.

Let $k$ be a field and $Q \in k[x, y, z]$ be a homogeneous polynomial of degree $a$. We denote by $\mathbb{C}_{Q} \subset \mathbb{P}^{2}$ the subvariety of 1 -dimensional subspaces $L$ in $\mathbb{A}^{3}$, such that $Q_{L} \equiv 0$, and write $C_{Q}:=\mathbb{C}_{Q}(k)$.

\section{Claim A.3.}

(1) The subvariety $\mathbb{C}_{Q} \subset \mathbb{P}^{2}$ is a curve, and therefore the subset $\mathbb{P}^{2}-\mathbb{C}_{Q}$ of $\mathbb{P}^{2}$ is Zariski dense.

(2) If $k=\mathbb{F}_{q}$ then $\left|C_{Q}\right| \leq a q$.

Proof. The part (1) is immediate. To prove (2) we fix a point $a \in \mathbb{P}^{2}(k)-C_{Q}$. For any line $M \subset \mathbb{P}^{2}(k)$ containing $a$, the subset $M \cap C_{Q} \subset M$ is defined by a non-zero polynomial of degree $a$. So $\left|M \cap C_{Q}\right| \leq a$. We see that $\left|C_{Q}\right| \leq a(q+1)$.

Let $R \in k[x, y, z]$ be a polynomial of degree $a$. We denote by $Q$ the degree $a$ homogeneous part of $P$. We denote by $\mathcal{L}_{3}$ the variety of affine lines $L$ in $\mathbb{A}^{3}$. For any $L \in \mathcal{L}_{3}$, we denote by $\tilde{L}$ the corresponding 1 -dimensional linear subspace of $\mathbb{A}^{3}$.

Let $\mathcal{L}_{R} \subset \mathcal{L}_{3}$ be the subvariety of lines $L$, such that $P_{L}$ is of degree $<a$.

\section{Claim A.4.}

(1) $L \in \mathcal{L}_{P} \leftrightarrow \tilde{L} \in C_{Q}$.

(2) $\left|\mathcal{L}_{P}\right| /|\mathcal{L}| \leq a / q$.

Proof. The part (1) is immediate and the part (2) follows from the part (1) and Claim A.3.

Let $k$ be a field and $\mathbb{X}:=\mathbb{X}_{\bar{P}}$, where $\bar{P}=\left(P_{i}\right)_{1 \leq i \leq c}$, are polynomials on $k^{N}$ of degrees $\leq d_{i}$. We denote by $\mathbb{A}$ the variety of affine lines in $\mathbb{X}$, by $\mathbb{B}$ the variety of 3-dimensional affine subspaces in $\mathbb{X}$ and by $\mathbb{E} \subset \mathbb{A}^{2}$ the subvariety of pairs if lines $L, L^{\prime} \in \mathbb{A}$ such that there exists unique $W \in \mathbb{B}$ containing both $L$ and $L^{\prime}$. We have natural projections $p_{1}, p_{2}: \mathbb{E} \rightarrow \mathbb{A}$ and the surjection $j: \mathcal{E} \rightarrow \mathcal{B}$. We write $A:=\mathbb{A}(k), B:=\mathbb{B}(k), E:=\mathbb{E}(k)$. For any subvariety $\mathbb{Z} \subset \mathbb{A}$ we define $\tilde{Z}:=p_{2}\left(p_{1}^{-1}(\mathbb{Z})\right)$.

Let $f: X \rightarrow k$ be a weakly polynomial function of degree $\leq a$. We denote by $C=C_{f} \subset A$ the subset of lines $L$ such that $\operatorname{deg}\left(f_{L}\right)=a$. For $W \in B$ we denote by $A(W) \subset A$ the set of lines $L \in W$, and write $C(W):=A(w) \cap C$. 
We will show that under the assumption that $r_{n c}(\bar{P}) \gg 1$ and $k$ is either a finite or an algebraically closed field, the assumption that $C$ is small implies that $C=\emptyset$.

We first consider the case when $k=\mathbb{F}_{q}$. In this case the size of $C$ is the number $|C|$. So the statement to prove is Proposition 4.37.

Proof. We start with the following result.

Claim A.5. $|C(W)| /|A(w)| \geq 1-a / q$ for any $W \in B$ containing some $L \in C$.

Proof. Since $f$ is weakly of degree $\leq d$, its restriction $f_{W}$ on $W$ is a polynomial of degree $\leq d$. Since $L \in C$, the restriction $f_{W}$ is of degree $d$ it follows from Claim A.4 that $|C(W)| /|A(w)| \geq 1-a / q$.

As follows from Lemma A.5 in [18] for any $\bar{d}=\left(d_{1}, \ldots, d_{c}\right)$ there exists $t(\bar{d})$ such that the subset $B \subset A \times A$ is $q^{-t(d)}$-dense for any $X=X_{\bar{P}}$, where $P$ is a polynomial of degree $d$. So it follows from Claim A.2 that in the case when $A(d, L, a)=t(d)+1$ and $a / q \leq 1 / 2$, we have $C=\emptyset$.

We consider now the case when $k$ is algebraically closed. In this case we measure the size of $C$ in terms of the codimension $\Delta(f)$ of the algebraic closure $\mathbb{Z}$ of $C \in \mathbb{A}$.

Proposition A.6. For any a there exists a constant $C(\bar{d}, a)$ such that the following holds.

Any weakly polynomial function $f$ on $X$ of degree $\leq$ a such that $\Delta(f) \geq C(\bar{d}, a)$ is actually of degree $<d$.

Proof. We start with the following result.

Claim A.7. For any $\bar{d}=\left(d_{1}, \ldots, d_{c}\right)$, there exists $t(\bar{d}, a)$, such that for any non-empty subvariety $\mathbb{Z} \subset \mathbb{A}$ of codimension $>t(\bar{d}, a)$ we have $\operatorname{dim}(\tilde{\mathbb{Z}})>\operatorname{dim}(\mathbb{Z})$.

Proof. It follows from Lemma A.5 in [18] (see Section 3.4) that $\operatorname{dim}\left(p_{1}^{-1}(L)\right) \geq$ $\operatorname{dim}(\mathcal{A})-t(\bar{d}, a)$. Now the same arguments as in the proof of Claim A.2 proves the inequality $\operatorname{dim}(\tilde{\mathbb{Z}})>\operatorname{dim}(\mathbb{Z})$.

Now we can finish the proof of Proposition A.6. Let $D \subset E$ be the set of pairs of lines $L, L^{\prime} \in E \cap(C \times C)$. As follows from Claim A.3 (1), the intersection $D \cap p_{1}^{-1}(L)(k)$ is dense in $p_{1}^{-1}(L)$. This implies that $\tilde{\mathbb{Z}}(k) \cap C$ is dense in $\tilde{\mathbb{Z}}$. Therefore $\tilde{\mathbb{Z}} \subset \mathbb{Z}$. It follows now from Claim A.7 that $\mathbb{Z}=\emptyset$ and therefore $C=\emptyset$.

\section{REFERENCES}

[1] Ananyan, T., Hochster, M. Strength conditions, small subalgebras, and Stillman bounds in degree $\leq 4$. arXiv: 1810.00413

[2] Alon, N., Kaufman, T., Krivelevich, M., Litsyn, S., Ron, D. Testing Low-Degree Polynomials over $G F(2)$. Approximation, Randomization, and Combinatorial Optimization. Algorithms and Techniques 188-199. 
[3] Bergelson, V.; Tao, T.; Ziegler, T. An inverse theorem for the uniformity seminorms associated with the action of $\mathbb{F}^{\infty}$. Geom. Funct. Anal. 19 (2010), no. 6, 1539-1596.

[4] Bhowmick A., Lovett S. Bias vs structure of polynomials in large fields, and applications in effective algebraic geometry and coding theory. arXiv:1506.02047

[5] Bik, A.; Draisma, J.; Eggermont, R. Polynomials and tensors of bounded strength. Preprint available Arxiv 1805.01816

[6] Gowers, T. A new proof of Szemerédi's theorem. Geom. Funct. Anal. 11, (2001) 465-588.

[7] Gowers, W. T.; Milićević, L. A quantitative proof for the $U^{4}$ norm over finite fields. arxiv 1712.00241

[8] Green, B., Tao, T. The distribution of polynomials over finite fields, with applications to the Gowers norms. Contrib. Discrete Math. 4 (2009), no. 2, 1-36.

[9] Hatami, H, Hatami, P. Lovett, S Higher order Fourier analysis and applications. Available at http://cseweb.ucsd.edu/slovett/files/survey-higher-order-fourier.pdf.

[10] Haramaty, E.; Shpilka, A. On the structure of cubic and quartic polynomials, STOC 2010.

[11] Hrushovski, E The Elementary Theory of the Frobenius Automorphisms arXiv: 0406514

[12] Janzer, O. Polynomial bound for the partition rank vs the analytic rank of tensorsLow analytic rank implies low partition rank for tensors. arXiv:1902.11207

[13] Jutla, C.S.; Patthak, A.C. ; Rudra, A. ; Zuckerman, D. Testing low-degree polynomials over prime fields. Random Structures and Algorithms Volume 35, Issue 2, 2009, Pages 163-193.

[14] Kaufman, T., Lovett, S. Worst case to average case reductions for polynomials. Proc. 49th Annual IEEE Symposium on Foundations of Computer Science (2008),166-175.

[15] Kaufman, T., Ron, D. Testing polynomials over general fields. SIAM J. on Comput., 36(3): 779-802, 2006.

[16] Kazhdan, D., Ziegler, T. Extending linear and quadratic functions from high rank varieties. arXiv:1712.01335

[17] Kazhdan, D., Ziegler, T. On ranks of polynomials. Algebr Represent Theor (2018) 21: 1017.

[18] Kazhdan, D., Ziegler, T. Polynomials as splines Sel. Math. New Ser. (2019) 25: 31.

[19] Kazhdan, D., Ziegler, T. Approximate cohomology. Sel. Math. New Ser. (2018) 24: 499.

[20] Kazhdan, D., Ziegler, T. On the codimension of the singular locus. arxiv.org/abs/1907.11750

[21] Lampert, A. Bias implies low rank for quartic polynomials. MSc. Thesis, October 2018. Available at arXiv:1902.10632.

[22] Lang, S., Weil , A. Number of points of varieties in finite fields. Amer. J. Math. 76 (1954)

[23] Lovett, S. The Analytic rank of tensors and its applications. Discrete Analysis 2019:7

[24] Lovett, S., Meshulam, R. , Samorodnitsky, A. Inverse conjecture for the Gowers norm is false, STOC 2008.

[25] Matsumura, H Commutative ring theory. Cambridge Studies in Advanced Mathematics, 8. Cambridge University Press, Cambridge, 1989.

[26] Milićević, L. Polynomial bound for partition rank in terms of analytic rank arXiv: 1902.09830

[27] W. Schmidt, The density of integer points on homogeneous varieties. Acta Math. 154 (1985), no. 3-4, 243296.

[28] Tao, T., Ziegler, T. The inverse conjecture for the Gowers norm over finite fields via the correspondence principle Analysis \& PDE Vol. 3 (2010), No. 1, 1-20.

[29] Tao,T.; Ziegler, T. The inverse conjecture for the Gowers norm over finite fields in low characteristic, Ann. Comb. 16 (1) (2012) 121-188. 
Einstein Institute of Mathematics, Edmond J. Safra Campus, Givaat Ram The Hebrew University of Jerusalem, Jerusalem, 91904, IsRAel

E-mail address: david.kazhdan@mail.huji.ac.il

Einstein Institute of Mathematics, Edmond J. Safra Campus, Givaat Ram The Hebrew University of Jerusalem, Jerusalem, 91904, Israel

E-mail address: tamarz@math.huji.ac.il 